\title{
TRACE SCALING AUTOMORPHISMS OF CERTAIN STABLE AF ALGEBRAS II
}

\author{
OLA BRATTELI AND AKITAKA KISHIMOTO
}

\begin{abstract}
Two automorphisms of a simple stable AF-algebra with a finite dimensional lattice of lower semicontinuous traces are shown to be outer conjugate if they act in the same way on $K_{0}$ and the extremal traces are scaled by numbers which are not equal to 1 and satisfy a certain condition (which holds if the scaling factors are all less than 1). The proof goes via the Rohlin property. As an application we consider the problem of classifying conjugacy or cocycle conjugacy classes of certain actions of $\mathbf{T}$ on a separable simple purely infinite $\mathrm{C}^{*}$-algebra.
\end{abstract}

\section{INTRODUCTION}

This paper is a continuation of [EEK] and [EK]. In the latter of these papers the case that $\mathfrak{A}$ is a stable $\mathrm{AF}$ algebra with totally ordered dimension group $K_{0}(\mathfrak{A})$ is considered. In this case $\mathfrak{A}$ has a densely defined lower semicontinuous trace $\tau$ (unique up to constant multiples) such that $K_{0}(\mathfrak{A})$ identifies with the range $\tau_{*}\left(K_{0}(\mathfrak{A})\right) \subset \mathbf{R}$, and in particular $K_{0}(\mathfrak{A})$ has no infinitesimal elements (i.e., $\operatorname{ker} \tau_{*}=$ $0)$. If $\alpha$ is an automorphism of $\mathfrak{A}$, there exists necessarily a $\lambda>0$ such that $\tau_{*} \alpha_{*}=\lambda \tau_{*}$, and it is proved in [EK] that if $\lambda \neq 1$ then $\alpha$ has the following Rohlin property: For any $k \in \mathbf{N}$, any projection $e \in \mathfrak{A}$, any finite subset $\mathcal{F}$ of $\mathfrak{A}$, and $\varepsilon>0$, there exists an orthogonal family $\left\{e_{i j} ; i=1,2 ; j=0,1, \ldots=k_{i}-1\right\}$ of projections in $\mathfrak{A}$ with $k_{1}=k, k_{2}=k+1$ such that

$$
\begin{aligned}
& \sum_{i=1}^{2} \sum_{j=0}^{k_{i}-1} e_{i j} \geq e, \\
& \left\|\alpha\left(e_{i j} e\right)-e_{i j+1} \alpha(e)\right\|<\varepsilon, \quad j=0,1, \ldots, k_{i}-2, \\
& \left\|\alpha\left(\left(e_{1 k_{1}-1}+e_{2 k_{2}-1}\right) e\right)-\left(e_{10}+e_{20}\right) \alpha(e)\right\|<\varepsilon, \\
& \left\|\left[x, e_{i j}\right]\right\|<\varepsilon, \quad x \in \mathcal{F},
\end{aligned}
$$

where $e_{i k_{i}}=e_{i 0}$. Here we may further impose the condition $\left[e_{i j}, e\right]=0$.

(This is slightly different from the Rohlin property given in 2.1 of [EK], but this version easily follows and is strong enough to prove 3.1 of [EK], i.e., the stability of $\alpha$. See also [K98], where a relaxation of this kind is discussed.)

It was also established in [EK] that if $\mathfrak{A}$ is any $\mathrm{AF}$ algebra and $\alpha, \beta$ are automorphims of $\mathfrak{A}$ with the Rohlin property, then if $\alpha_{*}=\beta_{*}$ on $K_{0}(\mathfrak{A}), \alpha$ and $\beta$ are outer conjugate: For any $\varepsilon>0$ there is an automorphism $\sigma$ of $\mathfrak{A}$ and a unitary $U$ in $\mathfrak{A}+\mathbb{1}$ such that $\alpha=\operatorname{Ad} U \circ \sigma \circ \beta \circ \sigma^{-1},\|U-\mathbb{1}\|<\varepsilon$ and $\sigma_{*}=$ id, so the previous theorem has an immediate corollary. The aim of the present paper is to prove the Rohlin property for trace scaling automorphisms under less stringent assumptions on $\mathfrak{A}$. Instead of assuming that $\mathfrak{A}$ has one trace separating elements in $K_{0}(\mathfrak{A})$ we will assume that the set of lower semicontinuous traces on $\mathfrak{A}$ form a finite dimensional 
lattice, i.e. if $E$ is a fixed projection in the simple stable AF-algebra $\mathfrak{A}$ then $\{\tau \mid \tau$ is a trace on $\mathfrak{A}$ and $\tau(E)=1\}$ is a finite-dimensional simplex. In order to prove the Rohlin property of an automorphism $\alpha$, we must assume a scaling property of the action $\alpha_{*}$ of $\alpha$ on the lattice of traces: As $\alpha_{*}$ maps extremal traces into extremal traces, $\alpha_{*}$ permutes the finite number of extremal rays in the space of traces, and hence some power of $\alpha_{*}$ scales all the extremal traces. We choose the smallest positive integer $p$ such that $\alpha_{*}^{p}$ has this property and let $\Lambda$ be the set of scales;

$$
\Lambda=\left\{\lambda \mid \alpha_{*}^{p}(\tau)=\tau \alpha^{p}=\lambda \tau \text { for an extreme trace } \tau\right\} .
$$

This is a finite subset of $(0, \infty)$.

Definition 1.1. If $\mathfrak{A}$ is a stable simple AF-algebra such that the set of lower semicontinuous traces form a finite dimensional lattice, and $\alpha$ is an automorphism of $\mathfrak{A}$, we say that $\alpha$ scales the traces if no extremal trace $\tau$ is invariant under any nonzero power $\alpha^{n}$ of $\alpha$. The set $\Lambda$ of positive numbers introduced prior to the definition is called the set of scales for $\alpha$, and thus $\alpha$ scales the traces if and only if the set of scales does not contain 1 .

To prove the main result in this paper we need more than that $\alpha$ scales the traces. The result is:

Theorem 1.2. Let $\mathfrak{A}$ be a simple stable AF algebra such that the (densely defined) lower semicontinuos traces form a finite dimensional lattice. Assume that $\alpha$ scales the traces, and moreover that the ring $\mathbf{Z}\left[x, x^{-1}\right]$ of polynominals in $x$ and $x^{-1}$ with integer coefficients is dense in the algebra $C(\Lambda)$ of real functions on the scales. It follows that $\alpha$ has the Rohlin property.

This theorem will be proved in Section 2.

Remark 1.3. The last condition in the theorem automatically implies that $1 \notin \Lambda$, i.e. that $\alpha$ scales the traces. The condition is fulfilled if there is an element $p \in$ $\mathbf{Z}\left[x, x^{-1}\right]$ such that $0<|p(\lambda)|<1$ for all $\lambda \in \Lambda$ by corollary 9.3 in $[$ Fer , and such a $p$ automatically exist if in addition to $1 \notin \Lambda$ one has $\Lambda \subset(0,2), \Lambda \subset\left(\frac{1}{2}, \infty\right)$, or $\Lambda \subset \mathbf{Q}$. There is a finite set $\Lambda$ for which this condition is not fulfilled. See $2.8-9$ below and [Fer] for details.

We show in Example 2.10 below how to construct $\mathrm{C}^{*}$-dynamical systems satisfying the hypotheses of Theorem 1.2 for a given $\Lambda$.

Corollary 1.4. Let $\mathfrak{A}$ be a simple stable AF algebra such that the lower semicontinous traces form a finite dimensional lattice. Assume that the set $\Lambda$ of scales of $\alpha$ satisfies the condition in theorem 1.2. Let $\beta$ be another automorphism of $\mathfrak{A}$ such that $\beta_{*}=\alpha_{*}$ on $K_{0}(\mathfrak{A})$. Then for any $\varepsilon>0$ there is an automorphism $\sigma$ of $\mathfrak{A}$ and a unitary $U$ in $\mathfrak{A}+\mathbb{1}$ such that $\alpha=\operatorname{Ad} U \circ \sigma \circ \beta \circ \sigma^{-1},\|U-\mathbb{1}\|<\varepsilon$ and $\sigma_{*}=$ id.

Proof. This follows from Theorem 1.2 and EK, Theorem 4.1].

By combining this with a result of Krieger Kri, we obtain a criterion for outer conjugacy of automorpisms of stationary systems [Eff, [Tor]. To define these, let $\varphi$ be a $r \times r$ matrix with nonnegative integer matrix elements, and let $G(\varphi)$ be the ordered abelian group defined as the inductive limit of

$$
\mathbf{Z}^{r} \stackrel{\varphi}{\longrightarrow} \mathbf{Z}^{r} \stackrel{\varphi}{\longrightarrow} \mathbf{Z}^{r} \rightarrow
$$

where each $\mathbf{Z}^{r}$ is ordered by requiring non-negativity of the $r$ coordinates. There is a unique stable $\mathrm{AF}$ algebra $\mathfrak{A}_{\varphi}$ associated to the dimension group $G(\varphi)$, and if 
$\varphi$ is primitive in the sense that $\varphi^{n}$ has strictly positive matrix elements for some $n \in \mathbf{N}$, the algebra $\mathfrak{A}_{\varphi}$ is simple with a trace $\tau_{\varphi}$ which is unique up to a scale. In fact, if $\lambda_{\varphi}$ is the Perron-Frobenius eigenvalue of $\varphi$ and $\eta_{\varphi}$ is a corresponding left eigenvector,

$$
\eta_{\varphi} \varphi=\lambda_{\varphi} \eta_{\varphi}
$$

the trace $\tau_{\varphi}$ is given on the $n^{\prime}$ th group $\mathbf{Z}^{r}$ as

$$
g \rightarrow \lambda_{\varphi}^{-n}\left\langle\eta_{\varphi} \mid g\right\rangle
$$

Now, let $\sigma_{\varphi^{*}}$ be the natural shift automorphism of $G(\varphi)$ determined by the inductive limit diagram (see [Eff, p. 37]). Then

$$
\tau_{\varphi^{*}} \circ \sigma_{\varphi^{*}}=\lambda_{\varphi}^{-1} \tau_{\varphi^{*}}
$$

Unless $r=1$ and $\varphi=1$, we have $\lambda_{\varphi}>1$. Let $\sigma_{\varphi}$ be some automorphism of $\mathfrak{A}_{\varphi}$ such that $\sigma_{\varphi^{*}}$ is the shift automorphism of $G(\varphi)$ defined above. For completeness we incorporate Krieger's result in the following corollary.

Corollary 1.5. Let $\varphi, \psi$ be primitive square matrics of dimension $r, s$, respectively with non-negative integer matrix units and assume $(\varphi, r) \neq(1,1)$. Let $G(\varphi), G(\psi)$ be the assosiated dimension groups $G(\varphi), G(\psi)$, respectively, and let $\sigma_{\varphi}, \sigma_{\psi}$ be associated automorphisms of the corresponding stable AF algebras $\mathfrak{A}_{\varphi}, \mathfrak{A}_{\psi}$. Then the following conditions are equivalent:

(1) $\varphi$ and $\psi$ are shift equivalent, i.e. there exists $k \in \mathbf{N}$ and there exists nonnegative rectangular $r \times s$ (resp. $s \times r$ ) matrices $A, B$ such that

$$
\begin{aligned}
& A \psi=\varphi A, B \varphi=\psi B \\
& A B=\varphi^{k}, B A=\psi^{k}
\end{aligned}
$$

(2) $\left(G(\varphi), \sigma_{\varphi^{*}}\right)$ and $\left(G(\psi), \sigma_{\psi^{*}}\right)$ are isomorphic, i.e. there exists an order isomorphism $\gamma_{*}: G(\psi) \rightarrow G(\varphi)$ with $\gamma_{*} \circ \sigma_{\psi *}=\sigma_{\varphi^{*}} \circ \gamma_{*}$

(3) The $C^{*}$-dynamical systems $\left(\mathfrak{A}_{\psi}, \sigma_{\psi}\right)$ and $\left(\mathfrak{A}_{\varphi}, \sigma_{\varphi}\right)$ are outer conjugate in the sense that there exists an isomorphism $\gamma: \mathfrak{A}_{\psi} \rightarrow \mathfrak{A}_{\varphi}$ and a unitary $U$ in $\mathfrak{A}_{\varphi}+\mathbb{1}$ such that $\sigma_{\varphi}=\operatorname{Ad} U \circ \gamma \circ \sigma_{\psi} \circ \gamma^{-1}$.

Proof. (1) $\Leftrightarrow(2)$ is in [Kri, $\S 4.2]$, see also [Eff. Theorem 6.4].

$(2) \Rightarrow(3)$ follows from Corollary 1.4 , and $(3) \Rightarrow(2)$ is trivial, by defining $\gamma_{*}$ from $\gamma$.

Remark 1.6. The assumption $(\varphi, r) \neq(1,1)$ is irrelevant for the equivalence $(1) \Leftrightarrow$ (2). It is inserted to assume that the Perron-Frahenius eigenvalue is larger than 1 , such that $\sigma_{\varphi}$ really scales the trace by a scale less than 1 . Note that $(2) \Rightarrow(3)$ actually is false in this "trivial" case, where $G=\mathbf{Z}$ and $\sigma_{\varphi^{*}}=\iota$ thus $\mathfrak{A}_{\varphi}=\mathfrak{A}_{\psi}=$ the algebra of compact operators. If we take $\sigma_{\psi}=$ id and $\sigma_{\varphi}=\operatorname{Ad}(V)$, where $V-\mathbb{1}$ is non-compact, it is clear that $\sigma_{\varphi}$ and $\sigma_{\psi}$ is not related as in (3) (Of course the Rohlin property also fails).

Remark 1.7. Note more generally that the condition of finite-dimensionality of the lattice of traces always is fulfilled if $\mathfrak{A}=\overline{\bigcup_{n} \mathfrak{A}_{n}}$ is an AF algebra, and the dimension of the center of $\mathfrak{A}_{n}$ in the generating sequence is bounded in $n$ by a constant $K$. The projective dimension of the lattice of traces is then at most $K-1$. This is because the restriction of a trace to any $\mathfrak{A}_{n}$ is a trace.

In the situation of Theorem 1.2 it follows from [R2] that the crossed product $\mathfrak{A} \times \mathbf{Z}$ is purely infinite. As an application of Theorem 1.2 we will in Section 4 discuss 
conjugacy and cocycle conjugacy classes of certain actions of $\mathbf{T}$ on a separable simple purely infinite $\mathrm{C}^{*}$-algebra (whose crossed product is $\mathrm{AF}$ ).

Let us end the introduction by mentioning that a result analoguos to Corollary 1.4 has been proved by H. Nakamura recently for the infinite case Nak; If two automorpisms of a nuclear separable simple purely infinite $\mathrm{C}^{*}$-algebra defines the same $K K$ class and have the Rohlin property, they are outer conjugate. (A similar result in the $A \mathbf{T}$ case is also found in [K98].) The expected characterization for the Rohlin property has been shown by M. Izumi Nak i.e., if $\mathfrak{A}$ is a separable purely infinite simple $\mathrm{C}^{*}$-algebra, and $\alpha$ is an automorphism of $\mathfrak{A}$ such that $\alpha^{n}$ is outer for $n \neq 0$, then $\alpha$ has the Rohlin property. The proof goes roughly by lifting $\alpha$ to the $\mathrm{C}^{*}$-algebra of central sequences modulo trivial central sequences, which is known to be purely infinite [KP], and then prove the Rohlin property there without worrying about centrality.

\section{Proof of Theorem 1.2}

If $\alpha_{*}$ denotes the action of $\alpha$ on $K_{0}(\mathfrak{A})$, then $K_{0}(\mathfrak{A})$ is a $\mathbf{Z}\left[x, x^{-1}\right]$ module as follows: If $p\left(x, x^{-1}\right) \in \mathbf{Z}\left[x, x^{-1}\right]$ and $g \in K_{0}(\mathfrak{A})$, define the action of $p$ on $g$ by

$$
p g=p\left(\alpha_{*}, \alpha_{*}^{-1}\right) g .
$$

In the proof of Theorem 1.2 we will use the fact that $\mathbf{Z}\left[x, x^{-1}\right] g$ is sufficiently big for each $g \in K_{0}(\mathfrak{A})_{+} \backslash\{0\}$. The condition on the scaling factors $\Lambda$ is imposed for this purpose (cf. 2.7).

In proving Theorem 1.2 we will closely follow the method in Section 2 of EK and recall the Rohlin property as defined there:

For any $k \in \mathbf{N}$ there are positive integers $k_{1}, \ldots, k_{m} \geq k$ such that for any projections $E, e$ in $\mathfrak{A}$, any unitary $U \in \mathfrak{A}+\mathbf{C} \mathbb{1}$, any finite subset $\mathcal{F}$ in $\mathfrak{A}_{E}=E \mathfrak{A} E$ and any $\varepsilon>0$ with $e \leq E, \operatorname{Ad} U \circ \alpha(e) \leq E, e \in \mathcal{F}, \operatorname{Ad} U \circ \alpha(e) \in \mathcal{F}$ there exists a family $\left\{e_{i, j} \mid i=1, \ldots, m, j=0, \ldots, k_{i}-1\right\}$ of projections in $\mathfrak{A}$ such that

$$
\begin{gathered}
\sum_{i} \sum_{j} e_{i, j}=E \\
\left\|\operatorname{Ad} U \circ \alpha\left(e_{i, j} e\right)-e_{i, j+1} \operatorname{Ad} U \circ \alpha(e)\right\|<\varepsilon \\
\left\|\left[x, e_{i, j}\right]\right\|<\varepsilon
\end{gathered}
$$

for $i=1, \ldots, m, j=0, \ldots, k_{i}-1$ and $x \in \mathcal{F}$, where $e_{i, k_{i}}=e_{i, 0}$. (Here we can take $m=2, k_{1}=k$, and $k_{2}=k+1$.) Note that we have imposed the condition

$$
\left\|\operatorname{Ad} U \circ \alpha\left(e_{i, k_{i}-1} e\right)-e_{i, 0} \operatorname{Ad} U \circ \alpha(e)\right\|<\varepsilon
$$

for each $i$, which is stronger than the corresponding part of the Rohlin property introduced in Section 1:

$$
\left\|\operatorname{Ad} U \circ \alpha\left(\left(\sum_{i} e_{i, k_{i}-1}\right) e\right)-\left(\sum_{i} e_{i, 0}\right) \operatorname{Ad} U \circ \alpha(e)\right\|<\varepsilon .
$$

Our assumption on the set of scales will make it possible to prove the Rohlin property in this stronger form. (We anticipate that the weaker form of the Rohlin property stated in Section 1 could be true with the special assumption on the set $\Lambda$ of scales in Theorem 2.1. replaced by just $1 \notin \Lambda$. This weaker form of the Rohlin property suffices for the proof of Corollary 1.4.)

By making an arbitrarily small inner perturbation of $\alpha$ and choosing an increasing sequence $\mathfrak{A}_{n}$ of finite-dimensional subalgebras as a subsequence of any given 
such sequence, we may assume that

$$
\begin{aligned}
& \alpha^{-1}\left(\mathfrak{A}_{n}\right) \subseteq \mathfrak{A}_{n+1} \\
& \alpha\left(\mathfrak{A}_{n}\right) \subseteq \mathfrak{A}_{n+1}
\end{aligned}
$$

for all $n$ (and $\bigcup_{n} \mathfrak{A}_{n}$ is dense in $\mathfrak{A}$ ). Fix some non-zero projection $E$ in $\mathfrak{A}_{1}$. For given $n=1,2, \ldots$, let $\mathfrak{A}_{n}=\bigoplus_{j} \mathfrak{A}_{n, j}$ be the central decomposition of $\mathfrak{A}_{n}$ where $\mathfrak{A}_{n, j}$ is a full matrix algebra. Let $p_{j}^{(n)} \in \mathfrak{A}_{n} \cap \mathfrak{A}_{n}^{\prime}$ be the central projection corresponding to $\mathfrak{A}_{n, j}$. By simplicity of $\mathfrak{A}$, we have either $p_{j}^{(n)} E=0$ (this only happens when $n$ is small), or

$$
K_{0}\left(\mathfrak{A} p_{j}^{(n)} E\right) \simeq K_{0}(\mathfrak{A})
$$

Since $\alpha\left(\mathfrak{A}_{n}\right) \subseteq \mathfrak{A}_{n+1}, \alpha^{-1}\left(\mathfrak{A}_{n}\right) \subseteq \mathfrak{A}_{n+1}$ and

$$
[\alpha(x), y]=\alpha\left(\left[x, \alpha^{-1} y\right]\right)
$$

we have

$$
\alpha\left(\mathfrak{A} \cap \mathfrak{A}_{n}^{\prime}\right) \subseteq \mathfrak{A} \cap \mathfrak{A}_{n-1}^{\prime}
$$

for all $n$.

Now pick a projection $e$ in some $\mathfrak{A}_{n}$ such that $e \leq E$ and $[\alpha(e)] \leq[E]$ and the central support of $e$ in $\mathfrak{A}_{n E}$ is $E$. By replacing $n$ by a larger $n$ and modifying $\alpha$ by an inner automorphism $\operatorname{Ad} U$ with $U \in \bigcup_{k} \mathfrak{A}_{k}+\mathbf{C} \mathbb{1}$ we may also assume that Ad $U \circ \alpha(e) \leq E$ and the central support of $\operatorname{Ad} U \circ \alpha(e)$ in $\mathfrak{A}_{n E}$ is $E$. Replacing $\alpha$ by $\operatorname{Ad} U \circ \alpha$ and relabeling the algebras $\mathfrak{A}_{n}$ we may thus assume

$$
\begin{gathered}
e, \alpha(e), E \in \mathfrak{A}_{1}, \\
e \leq E \quad \alpha(e) \leq E,
\end{gathered}
$$

Central support of $e$ and $\alpha(e)$ in $\mathfrak{A}_{1 E}$ (and thus in all $\mathfrak{A}_{n E}$ ) is $E$.

We now define a unital endomorphism.

$$
\widetilde{\alpha}: E\left(\mathfrak{A} \cap \mathfrak{A}_{n}^{\prime}\right) E \rightarrow E\left(\mathfrak{A} \cap \mathfrak{A}_{n-1}^{\prime}\right) E
$$

as follows: Because of simplicity of $\mathfrak{A}$ and the central support properties of $e, \alpha(e)$ we have

and

$$
E\left(\mathfrak{A} \cap \mathfrak{A}_{n}^{\prime}\right) E \cong e\left(\mathfrak{A} \cap \mathfrak{A}_{n}^{\prime}\right) e
$$

$$
E\left(\mathfrak{A} \cap \mathfrak{A}_{n-1}^{\prime}\right) E \cong \alpha(e)\left(\mathfrak{A} \cap \mathfrak{A}_{n-1}^{\prime}\right) \alpha(e), \quad n \geq 2
$$

and since $\alpha$ maps $e\left(\mathfrak{A} \cap \mathfrak{A}_{n}^{\prime}\right) e$ into $\alpha(e)\left(\mathfrak{A} \cap \mathfrak{A}_{n-1}^{\prime}\right) \alpha(e)$, we get a morphism

$$
\widetilde{\alpha}: E\left(\mathfrak{A} \cap \mathfrak{A}_{n}^{\prime}\right) E \rightarrow E\left(\mathfrak{A} \cap \mathfrak{A}_{n-1}^{\prime}\right) E
$$

by the requirement that the following diagram is commutative:

$$
\begin{array}{cccc}
E(\mathfrak{A} & \left.\cap \mathfrak{A}_{n}^{\prime}\right) E & \stackrel{\widetilde{\alpha}}{\longrightarrow} & E\left(\mathfrak{A} \cap \mathfrak{A}_{n-1}^{\prime}\right) E \\
\downarrow & \stackrel{ }{\downarrow} & & \cong \\
e\left(\mathfrak{A} \cap \mathfrak{A}_{n}^{\prime}\right) e & \stackrel{\alpha}{\longrightarrow} & \alpha(e)\left(\mathfrak{A} \cap \mathfrak{A}_{n-1}^{\prime}\right) \alpha(e)
\end{array}
$$

Let us explain the vertical isomorphisms, for example the one to the left: As $e \in$ $\mathfrak{A}_{1 E} \subseteq \mathfrak{A}_{n E}$ has central support $E$ in $\mathfrak{A}_{n E}$, it follows that $e$ has nonzero product with each of the minimal central projections $p_{j}^{(n)} E$ in $\mathfrak{A}_{n E}$ in $\mathfrak{A}_{n E}$. Let $\left(e_{k, l}^{(n, j)}\right)_{1 \leq k, l \leq[n, j]}$ be a complete set of matrix units for $\left(\mathfrak{A}_{n, j}\right)_{E}$. There is a projection of $\overline{\mathfrak{A}}_{E}$ onto $\left(\mathfrak{A} \cap \mathfrak{A}_{n}^{\prime}\right)_{E}$ given by

$$
x \longrightarrow \sum_{j, k} e_{k, 1}^{(n, j)} x e_{1, k}^{(n, j)}
$$


and as $e_{1, k}^{(n, j)} p_{i}^{(n)}=\delta_{i, j} e_{1, k}^{(n, j)}$ it follows that

$$
\begin{aligned}
\left(\mathfrak{A} \cap \mathfrak{A}_{n}^{\prime}\right) p_{j}^{(n)} E & =\left(\mathfrak{A} \cap \mathfrak{A}_{n, j}^{\prime}\right) p_{j}^{(n)} E \\
& \cong e_{1,1}^{(n, j)} \mathfrak{A} e_{1,1}^{(n, j)}
\end{aligned}
$$

Since $e$ has central support $E$, we may choose the matrix units $\left(e_{k, l}^{(n, j)}\right)$ such that $e_{1,1}^{(n, j)} \leq e p_{j}^{(n)}$ and $e p_{j}^{(n)}$ is the sum of the $e_{i, i}^{(n, j)}$ it has nonzero product with. Thus repeating the argument above with $E$ replaced by $e$, we deduce

$$
\left(\mathfrak{A} \cap \mathfrak{A}_{n}^{\prime}\right) p_{j}^{(n)} e \cong e_{1,1}^{(n, j)} \mathfrak{A} e_{1,1}^{(n, j)}
$$

we thus obtain the left vertical isomorphism.

We shall prove below that $\widetilde{\alpha}$, as a partially defined endomorphism of $\mathfrak{A}_{E}$, has the usual Rohlin property.

Note that $\widetilde{\alpha}$ defines a morphism from $E\left(\mathfrak{A} \cap \mathfrak{A}_{n}^{\prime}\right) p_{j}^{(n)} E$ into $E\left(\mathfrak{A} \cap \mathfrak{A}_{n-1}^{\prime}\right) E$ by restriction and since $p_{i}^{(n-1)} \in\left(\mathfrak{A} \cap \mathfrak{A}_{n-1}^{\prime}\right)^{\prime}, \widetilde{\alpha}$ defines a morphism

$$
\widetilde{\alpha}(i, j): E\left(\mathfrak{A} \cap \mathfrak{A}_{n}^{\prime}\right) p_{j}^{(n)} E \longrightarrow E\left(\mathfrak{A} \cap \mathfrak{A}_{n-1}^{\prime}\right) p_{i}^{(n-1)} E
$$

by multiplying $\widetilde{\alpha}(x)$ with $p_{i}^{(n-1)}$. Let

$$
\widetilde{\alpha}_{*}(i, j): K_{0}\left(\left(\mathfrak{A} \cap \mathfrak{A}_{n}^{\prime}\right) p_{j}^{(n)} E\right) \rightarrow K_{0}\left(\left(\mathfrak{A} \cap \mathfrak{A}_{n-1}^{\prime}\right) p_{i}^{(n-1)} E\right)
$$

be the corresponding map of the $K_{0}$-groups. Recall that

$$
\left(\mathfrak{A} \cap \mathfrak{A}_{n}^{\prime}\right) p_{j}^{(n)} E=\left(\mathfrak{A} \cap \mathfrak{A}_{n, j}^{\prime}\right) p_{j}^{(n)} E \cong e_{1,1}^{(n, j)} \mathfrak{A} e_{1,1}^{(n, j)} .
$$

Since $\mathfrak{A}$ is a simple AF algebra, we deduce that

$$
K_{0}\left(\left(\mathfrak{A} \cap \mathfrak{A}_{n}^{\prime}\right) p_{j}^{(n)} E\right) \cong K_{0}\left(e_{1,1}^{(n, j)} \mathfrak{A} e_{1,1}^{(n, j)}\right) \cong K_{0}(\mathfrak{A}) .
$$

Let us denote this isomorphism by $I_{*}^{(n, j)}$,

$$
I_{*}^{(n, j)}: K_{0}\left(\left(\mathfrak{A} \cap \mathfrak{A}_{n}^{\prime}\right) p_{j}^{(n)} E\right) \stackrel{\cong}{\longrightarrow} K_{0}(\mathfrak{A})
$$

This should be distinguished from the monomorphism

$$
\iota_{*}^{(n, j)}: K_{0}\left(\left(\mathfrak{A} \cap \mathfrak{A}_{n}^{\prime}\right) p_{j}^{(n)} E\right) \rightarrow K_{0}(\mathfrak{A})
$$

which comes from the embedding

$$
\left(\mathfrak{A} \cap \mathfrak{A}_{n}^{\prime}\right) p_{j}^{(n)} E \subseteq \mathfrak{A}
$$

In fact if $\left(\mathfrak{A}_{n, j}\right)_{E}=M_{[n, j]}$ then

$$
\iota_{*}^{(n, j)}=[n, j] I_{*}^{(n, j)}
$$

and $K_{0}\left(\left(\mathfrak{A} \cap \mathfrak{A}_{n}^{\prime}\right) p_{j}^{(n)} E\right)$ identifies on the one side with a subgroup of $K_{0}(\mathfrak{A})$ (through $\left.\iota_{*}^{(n, j)}\right)$ and on the other side is isomorphic to $K_{0}(\mathfrak{A})\left(\right.$ through $\left.I_{*}^{(n, j)}\right)$.

Thus $\widetilde{\alpha}_{*}(i, j): K_{0}\left(\left(\mathfrak{A} \cap \mathfrak{A}_{n}^{\prime}\right) p_{j}^{(n)} E\right) \rightarrow K_{0}\left(\left(\mathfrak{A} \cap \mathfrak{A}_{n-1}^{\prime}\right) p_{i}^{(n-1)} E\right)$ defines maps $\widehat{\alpha}_{*}(i, j): K_{0}(\mathfrak{A}) \rightarrow K_{0}(\mathfrak{A})$ by $\widehat{\alpha}_{*}(i, j)=I_{*}^{(n-1, i)} \widetilde{\alpha}_{*}(i, j)\left(I_{*}^{(n, j)}\right)^{-1}$.

Lemma 2.1. The map $\alpha_{*}: K_{0}(\mathfrak{A}) \rightarrow K_{0}(\mathfrak{A})$ and the maps $\widehat{\alpha}_{*}(i, j): K_{0}(\mathfrak{A}) \rightarrow$ $K_{0}(\mathfrak{A})$ do all commute: $\widehat{\alpha}_{*}(i, j) \alpha_{*}=\alpha_{*} \widehat{\alpha}_{*}(i, j)$. 
Proof. We want to prove

$$
I_{*}^{(n-1, i)} \widetilde{\alpha}_{*}(i, j)\left(I_{*}^{(n, j)}\right)^{-1} \alpha_{*}=\alpha_{*} I_{*}^{(n-1, i)} \widetilde{\alpha}_{*}(i, j)\left(I_{*}^{(n, j)}\right)^{-1} .
$$

Multiplying to the left by $\left(I^{(n-1, i)}\right)^{-1}$ and to the right by $I^{(n, j)}$, this amounts to showing $\widetilde{\alpha}_{*}(i, j)\left(\left(I_{*}^{(n, j)}\right)^{-1} \alpha_{*} I_{*}^{(n, j)}\right)=\left(\left(I_{*}^{(n-1, i)}\right)^{-1} \alpha_{*} I_{*}^{(n-1, i)}\right) \widetilde{\alpha}_{*}(i, j)$ as a map from $K_{0}\left(\left(\mathfrak{A} \cap \mathfrak{A}_{n}^{\prime}\right) p_{j}^{(n)} E\right)$ into $K_{0}\left(\left(\mathfrak{A} \cap \mathfrak{A}_{n-1}^{\prime}\right) p_{i}^{(n-1)} E\right)$. To this end, pick a

$$
g \in K_{0}\left(\left(\mathfrak{A} \cap \mathfrak{A}_{n}^{\prime}\right) p_{j}^{(n)} E\right)
$$

such that

$$
0 \leq g, \alpha_{*}^{(n, j)}(g) \leq\left[p_{j}^{(n)} E\right]
$$

where $\alpha_{*}^{(n, j)}=\left(I_{*}^{(n, j)}\right)^{-1} \alpha_{*} I_{*}^{(n, j)}=\left(\iota_{*}^{(n, j)}\right)^{-1} \alpha_{*} \iota_{*}^{(n, j)}$ and $[\cdot]$ here refers to $K_{0}$-class in $\left(\mathfrak{A} \cap \mathfrak{A}_{n}^{\prime}\right) p_{j}^{(n)} E$. It suffices to verify the commutation on these $g^{\prime} s$ since they generate $K_{0}\left(\left(\mathfrak{A} \cap \mathfrak{A}_{n}^{\prime}\right) p_{j}^{(n)} E\right)$. Pick projections $h, f \in\left(\mathfrak{A} \cap \mathfrak{A}_{n}^{\prime}\right) p_{j}^{(n)} E$ such that

$$
g=[h] \quad \alpha_{*}^{(n, j)}(g)=[f]
$$

We may and will assume that $h, f \in \bigcup_{m=1}^{\infty} \mathfrak{A}_{m}$. Note that

$$
[\alpha(h)]_{K_{0}(\mathfrak{A})}=\alpha_{*} \iota_{*}^{(n, j)}(g)
$$

and

$$
\begin{aligned}
{[f]_{K_{0}(\mathfrak{A})} } & =\iota_{*}^{(n, j)}([f]) \\
& =\iota_{*}^{(n, j)} \alpha_{*}^{(n, j)}(g) \\
& =\alpha_{*} \iota_{*}^{(n, j)}(g) .
\end{aligned}
$$

If $\tau_{*}$ is any real-valued additive character on $K_{0}(\mathfrak{A})$ (no positivity assumed), there is a unique linear functional $\tau$ on $\bigcup_{m=1}^{\infty} \mathfrak{A}_{m}$ corresponding to $\tau_{*}$, which restricts to a scalar multiple of the standard trace on each $\mathfrak{A}_{m, k}$, such that

$$
\tau_{*}([p])=\tau(p)
$$

for each projection $p$ in $\bigcup_{m=1}^{\infty} \mathfrak{A}_{m}$. To show our commutation, we just need to show

$$
\begin{aligned}
\tau_{*} \iota_{*}^{(n-1, i)} \widetilde{\alpha}_{*}(i, j)([f]) \\
\quad=\tau_{*} \iota_{*}^{(n-1, i)} \alpha_{*}^{(n-1, i)} \widetilde{\alpha}_{*}(i, j)(g) \\
\quad=\tau_{*} \alpha_{*} \iota_{*}^{(n-1, i)} \widetilde{\alpha}_{*}(i, j)(g) \\
\quad=\tau \circ \alpha\left(\widetilde{\alpha}(h) p_{i}^{(n-1)}\right)
\end{aligned}
$$


But this follows from the computation (where we use $\mathfrak{A}_{n-1, i}$ to denote $\mathfrak{A}_{n-1, i} E$ )

$$
\begin{aligned}
\tau_{*} \iota_{*}^{(n-1, i)}\left(\widetilde{\alpha}_{*}(i, j)[f]\right) \\
=\tau\left(\widetilde{\alpha}(f) p_{i}^{(n-1)}\right) \\
=\frac{\tau\left(\alpha(f e) p_{i}^{(n-1)}\right)}{\operatorname{tr}_{\mathfrak{A}_{n-1, i}}(\alpha(e))} \\
=\tau \circ \alpha(f) \frac{\operatorname{tr}_{\mathfrak{A}_{n, j}}\left(e \alpha^{-1}\left(p_{i}^{(n-1)}\right)\right)}{\operatorname{tr}_{\mathfrak{A}_{n-1, i}}(\alpha(e))} \\
=\tau \circ \alpha(\alpha(h)) \frac{\operatorname{tr}_{\mathfrak{A}_{n, j}}\left(e \alpha^{-1}\left(p_{i}^{(n-1)}\right)\right)}{\operatorname{tr}_{\mathfrak{A}_{n-1, i}}(\alpha(e))} \\
=(\tau \circ \alpha) \circ \alpha(h) \frac{\operatorname{tr}_{\mathfrak{A}_{n, j}}\left(e \alpha^{-1}\left(p_{i}^{(n-1)}\right)\right)}{\operatorname{tr}_{\mathfrak{A}_{n-1, i}}(\alpha(e))} \\
=\frac{\tau \circ \alpha\left(\alpha(h e) p_{i}^{(n-1)}\right)}{\operatorname{tr}_{\mathfrak{A}_{n-1, i}}(\alpha(e))} \\
=\tau \circ \alpha\left(\widetilde{\alpha}(h) p_{i}^{(n-1)}\right)
\end{aligned}
$$

Here the second equality follows from the definition of $\widetilde{\alpha}$, and the third from $\tau\left(\alpha(f e) p_{i}^{(n-1)}\right)=\tau \circ \alpha\left(f e \alpha^{-1}\left(p_{i}^{(n-1)}\right)\right)$ and the fact that $f \in\left(\mathfrak{A} \cap \mathfrak{A}_{n}^{\prime}\right) p_{j}^{(n)} E$ commutes with $e$ and $\alpha^{-1}\left(p_{i}^{(n-1)}\right)$, and $e$ commutes with $\alpha^{-1}\left(p_{i}^{(n-1)}\right) \subseteq \mathfrak{A}_{n-2}^{\prime}$ so $e \alpha^{-1}\left(p_{i}^{(n-1)}\right)$ is a projection in a tensor product complement of $f$.

As in the case of $\alpha$ (but simpler) the embedding

$$
E\left(\left(\mathfrak{A} \cap \mathfrak{A}_{n}^{\prime}\right) p_{j}^{(n)}\right) E \subseteq E\left(\mathfrak{A} \cap \mathfrak{A}_{n-1}^{\prime}\right) E
$$

defines morphisms

$$
\iota_{*}(i, j): K_{0}\left(\left(\mathfrak{A} \cap \mathfrak{A}_{n}^{\prime}\right) p_{j}^{(n)} E\right) \rightarrow K_{0}\left(\left(\mathfrak{A} \cap \mathfrak{A}_{n-1}^{\prime}\right) p_{i}^{(n-1)} E\right)
$$

by multiplying with $p_{i}^{(n-1)}$, and again $\iota_{*}(i, j)$ can also be viewed as maps $K_{0}(\mathfrak{A}) \rightarrow$ $K_{0}(\mathfrak{A})$.

Lemma 2.2. The maps $\iota_{*}(i, j)$ commute with $\alpha_{*}$ :

$$
\iota_{*}(i, j) \alpha_{*}=\alpha_{*} \iota_{*}(i, j)
$$

Proof. If $0 \leq g, \alpha_{*} g \leq\left[p_{j}^{(n)} E\right]$, choose again projections $h \in\left(\mathfrak{A} \cap \mathfrak{A}_{n, j}^{\prime}\right) p_{j}^{(n)} E$ and $f$ such that

$$
\begin{aligned}
& {[h]=g} \\
& {[f]=\alpha_{*} g}
\end{aligned}
$$

Here we have identified $K_{0}\left(\left(\mathfrak{A} \cap \mathfrak{A}_{n, j}^{\prime}\right) p_{j}^{(n)} E\right)$ with a subgroup of $K_{0}(\mathfrak{A})$ through $\iota_{*}^{(n, j)}$. Thus, for any real valued additive character $\tau$ on $K_{0}(\mathfrak{A})$ we have again

$$
\begin{gathered}
\tau\left(f p_{i}^{(n-1)}\right)=\tau(f) \operatorname{tr}_{\mathfrak{A}_{n, j}}\left(p_{i}^{(n-1)}\right) \\
=\tau_{*}\left(\alpha_{*}(g)\right) \operatorname{tr}_{\mathfrak{A}_{n, j}}\left(p_{i}^{(n-1)}\right) \\
=\tau \circ \alpha(h) \operatorname{tr}_{\mathfrak{A}_{n, j}}\left(p_{i}^{(n-1)}\right) \\
=\tau \alpha\left(h p_{i}^{(n-1)}\right)
\end{gathered}
$$


establishing the lemma.

Lemma 2.3. The morphisms

$$
\widetilde{\alpha}_{*}(i, j), \iota_{*}(i, j): K_{0}\left(\left(\mathfrak{A} \cap \mathfrak{A}_{n}^{\prime}\right) p_{j}^{(n)} E\right) \rightarrow K_{0}\left(\left(\mathfrak{A} \cap \mathfrak{A}_{n-1}^{\prime}\right) p_{i}^{(n-1)} E\right)
$$

satisfy:

$$
\sum_{j} \widetilde{\alpha}_{*}(i, j)\left(\left[p_{j}^{(n)} E\right]\right)=\left[p_{i}^{(n-1)} E\right]
$$

and

$$
\sum_{j} \iota_{*}(i, j)\left(\left[p_{j}^{(n)} E\right]\right)=\left[p_{i}^{(n-1)} E\right]
$$

Proof. By definition of $\widetilde{\alpha}(i, j)$ we have

$$
\widetilde{\alpha}(i, j)\left(p_{j}^{(n)} E\right)=\widetilde{\alpha}\left(p_{j}^{(n)} E\right) p_{i}^{(n-1)} E .
$$

But

$$
\begin{aligned}
\sum_{j} \widetilde{\alpha}\left(p_{j}^{(n)} E\right) & =\widetilde{\alpha}\left(\sum_{j} p_{j}^{(n)} E\right) \\
& =\widetilde{\alpha}(E)=E
\end{aligned}
$$

SO

$$
\begin{aligned}
\sum_{j} \widetilde{\alpha}(i, j)\left(p_{j}^{(n)} E\right) & =E p_{i}^{(n-1)} E \\
& =p_{i}^{(n-1)} E
\end{aligned}
$$

and hence

$$
\sum_{j} \widetilde{\alpha}_{*}(i, j)\left(\left[p_{j}^{(n)} E\right]\right)=\left[p_{i}^{(n-1)} E\right]
$$

Replacing $\widetilde{\alpha}$ by $\iota=\mathrm{id}$ in the reasoning above, we obtain the other identity.

Before continuing the proof we describe the action $\alpha_{*}$ on the lattice of traces in more details.

If $\mathbf{R}_{+} \tau_{k}, k=1, \ldots, d$ are the extreme rays of the lattice of densely defined lower semi-continuous traces, then as $\alpha_{*}$ maps extremal traces into extremal traces, $\alpha_{*}$ permutes the extreme rays and we can choose the labels $k$ such that $\alpha_{*}$ maps $\mathbf{R}_{+} \tau_{k}$ onto $\mathbf{R}_{+} \tau_{k+1}$ except for a set $1 \leq k_{1}<k_{2}<\cdots<k_{l}=d$ such that $\alpha_{*}\left(\mathbf{R}_{+} \tau_{k_{i}}\right)=$ $\mathbf{R}_{+} \tau_{k_{i-1}+1}$ for $i=1, \ldots, l$, where $k_{0}=0$. But then $\alpha_{*}^{k_{i}-k_{i-1}}\left(\tau_{k}\right)=\lambda_{i}^{k_{i}-k_{i-1}}\left(\tau_{k}\right)$ for $k_{i-1}<k \leq k_{i}$ for a unique positive number $\lambda_{i}$. If $p$ denotes the least common multiple of $k_{i}-k_{i-1}, i=1,2, \ldots, l$, then $\alpha_{*}^{p}\left(\tau_{k}\right)$ is proportional to $\tau_{k}$ for all $k$ and $p$ is the smallest positive integer with this property. Hence the set $\Lambda$ of scales for $\alpha$ is $\left\{\lambda_{i}^{p}: i=1,2, \ldots, l\right\}$.

To continue the proof we now normalize $\tau_{k}$ by $\tau_{k}(E)=1$, where $E \in \mathfrak{A}_{1}$ is fixed as before.

We view $\tau_{1}, \ldots, \tau_{d}$ as mutually disjoint extremal trace states on $\mathfrak{A}_{E}$, and the weak closures $\pi_{\tau_{i}}\left(\mathfrak{A}_{E}\right)^{\prime \prime}$ are all isomorphic to the hyperfinite $\mathrm{II}_{1}$ factor.

Let $\omega$ be a free ultrafilter on $\mathbf{N}$ and let $\mathfrak{A}_{E \omega}$ be the $\mathrm{C}^{*}$-algebra of central sequences, i.e. $\mathfrak{A}_{E \omega}$ consists of elements $\left(x_{n}\right)$ in $l^{\infty}\left(\mathbf{N}, \mathfrak{A}_{E}\right)$ such that $\lim _{n \rightarrow \omega} \| x_{n} a-$ $a x_{n} \|=0$ for all $a \in \mathfrak{A}_{E}$ modulo sequences $\left(x_{n}\right)$ such that $\lim _{n \rightarrow \omega}\left\|x_{n}\right\|=0$. Since $\mathfrak{A}=\overline{\bigcup_{n} \mathfrak{A}_{n}}$ where the $\mathfrak{A}_{n}$ are finite dimensional, it follows that for any $x=\left(x_{n}\right) \in$ 
$\mathfrak{A}_{E \omega}$ we can find an increasing sequence $k_{n}$ in $\mathbf{N}$ and $x_{n}^{\prime} \in\left(\mathfrak{A} \cap \mathfrak{A}_{k_{n}}^{\prime}\right)_{E}$ such that $k_{n} \rightarrow \infty$ and

$$
\lim _{n \rightarrow \omega}\left\|x_{n}-x_{n}^{\prime}\right\|=0
$$

Define a homomorphism $\widetilde{\alpha}_{\omega}$ of $\mathfrak{A}_{E \omega}$ into itself by

$$
\widetilde{\alpha}_{\omega}(x)=\left(\widetilde{\alpha}\left(x_{n}^{\prime}\right)\right)
$$

One now checks that $\widetilde{\alpha}_{\omega}$ is well defined in the sense that it is independent of the earlier choice of $e$ and $U$. Again following EK one defines $\widetilde{\alpha_{\omega}^{-1}}$ and verify $\widetilde{\alpha_{\omega}^{-1}} \circ \widetilde{\alpha}_{\omega}=\widetilde{\alpha}_{\omega} \circ \widetilde{\alpha_{\omega}^{-1}}=$ id, so $\widetilde{\alpha}_{\omega}$ is an automorphism of $\mathfrak{A}_{E \omega}$.

Now define tracial states $\tau_{i \omega}$ on $\mathfrak{A}_{E \omega}$ by

$$
\tau_{i \omega}(x)=\lim _{n \rightarrow \omega} \tau_{i}\left(x_{n}\right)
$$

for $x=\left(x_{n}\right) \in \mathfrak{A}_{E \omega}$. We next show that the $\tau_{i \omega}$ are permuted cyclically by $\widetilde{\alpha}_{\omega}$, i.e.

$$
\tau_{i \omega} \circ \widetilde{\alpha}_{\omega}=\tau_{i+1 \omega}
$$

except for

$$
\tau_{k_{i} \omega} \circ \widetilde{\alpha}_{\omega}=\tau_{k_{i-1}+1 \omega}
$$

We first show that if $\tau$ is any extremal trace state on $\mathfrak{A}_{E}$, i.e. an extremal trace on $\mathfrak{A}$ with $\tau(E)=1$, then

$$
\tau_{\omega} \circ \widetilde{\alpha}=(\tau(\alpha(E)))^{-1}(\tau \circ \alpha)_{\omega}
$$

For this, pick any central sequence $x=\left(x_{n}\right) \in \mathfrak{A}_{E \omega}$ where $x_{n} \in\left(\mathfrak{A} \cap \mathfrak{A}_{k_{n}}^{\prime}\right)_{E}$ for some non-decreasing sequences $k_{n}$ with $k_{n} \rightarrow \infty$. When $k_{n}>k$ with $U \in \mathfrak{A}_{k-1}+\mathbf{C} \mathbb{1}$ we have

$$
\tau\left(\widetilde{\alpha}\left(x_{n}\right)\right)=\sum_{i} \tau\left(\widetilde{\alpha}\left(x_{n}\right) p_{i}^{(k-1)} E\right)
$$

Now

$$
y \in E\left(\mathfrak{A} \cap \mathfrak{A}_{k}^{\prime}\right) E \rightarrow \frac{\tau\left(\widetilde{\alpha}(y) p_{i}^{(k-1)} E\right)}{\tau\left(p_{i}^{(k-1)} E\right)}
$$

is a trace state. We have

$$
\widetilde{\alpha}(y) \in E\left(\mathfrak{A} \cap \mathfrak{A}_{k-1}^{\prime}\right) E
$$

so

$$
\widetilde{\alpha}(y) p_{i}^{(k-1)} E \in E\left(\mathfrak{A} \cap \mathfrak{A}_{k-1}^{\prime}\right) p_{i}^{(k-1)} E .
$$

Now, $\widetilde{\alpha}(y) p_{i}^{(k-1)} E$ and $\operatorname{Ad} U \alpha(e) p_{i}^{(k-1)}$ lie in different tensor factors of $\mathfrak{A}_{E} p_{i}^{(k-1)}$ so that

$$
\begin{aligned}
\tau(\widetilde{\alpha}(y) & \left.\operatorname{Ad} U \alpha(e) p_{i}^{(k-1)}\right) \\
= & \tau\left(\widetilde{\alpha}(y) p_{i}^{(k-1)}\right) \operatorname{tr}_{\mathfrak{A}_{k-1 i, E}}(\operatorname{Ad} U \alpha(e)) \\
= & \tau\left(\widetilde{\alpha}(y) p_{i}^{(k-1)}\right) \frac{\tau\left(\operatorname{Ad} U \alpha(e) p_{i}^{(k-1)}\right)}{\tau\left(p_{i}^{(k-1)} E\right)}
\end{aligned}
$$

Thus, for large $n$,

$$
\tau\left(\widetilde{\alpha}\left(x_{n}\right)\right)=\sum_{i} \frac{\tau\left(p_{i}^{(k-1)} E\right) \tau\left(\widetilde{\alpha}\left(x_{n}\right) \operatorname{Ad} U \alpha(e) p_{i}^{(k-1)} E\right)}{\tau\left(\operatorname{Ad} U \alpha(e) p_{i}^{(k-1)}\right)}
$$

But

$$
\widetilde{\alpha}\left(x_{n}\right) \operatorname{Ad} U \alpha(e)=\operatorname{Ad} U \alpha\left(x_{n} e\right)
$$


so, using the trace property:

$$
\tau\left(\widetilde{\alpha}\left(x_{n}\right)\right)=\sum_{i} \frac{\tau\left(p_{i}^{(k-1)} E\right)}{\tau\left(p_{i}^{(k-1)} \alpha(e)\right)}(\tau \circ \alpha)\left(\alpha^{-1}\left(p_{i}^{(k-1)}\right) e x_{n}\right)
$$

Now, $\frac{\tau \circ \alpha}{\tau(\alpha(E))}$ is a factor state on $\mathfrak{A}_{E}$, and hence

$$
\begin{aligned}
\lim _{n \rightarrow \omega} & \frac{\tau \alpha\left(\alpha^{-1}\left(p_{i}^{(k-1)}\right) e x_{n}\right)}{\tau(\alpha(E))} \\
= & \frac{\tau \alpha\left(\alpha^{-1}\left(p_{i}^{(k-1)}\right) e\right)}{\tau(\alpha(E))} \cdot \frac{(\tau \circ \alpha)_{\omega}(x)}{\tau(\alpha(E))}
\end{aligned}
$$

and hence, by the previous relation, $\lim _{n \rightarrow \omega} \tau\left(\widetilde{\alpha}\left(x_{n}\right)\right)=\sum_{i} \frac{\tau\left(p_{i}^{(k-1)} E\right)}{\tau(\alpha(E))}(\tau \alpha)_{\omega}(x)=$ $\frac{(\tau \alpha)_{\omega}(x)}{\tau(\alpha(E))}$, or:

$$
\tau_{\omega} \circ \widetilde{\alpha}_{\omega}=(\tau(\alpha(E)))^{-1}(\tau \circ \alpha)_{\omega} .
$$

It follows immediately that the trace states $\tau_{i \omega}$ are permuted cyclically (without scaling) by $\widetilde{\alpha}_{\omega}$. The states $\tau_{i \omega}$ are trace states on $\mathfrak{A}_{E \omega}$ because $\varphi_{\omega}$ is a trace state on $\mathfrak{A}_{E \omega}$ for any state $\varphi$ on $\mathfrak{A}_{E}$, see $\mathrm{C74}$. Furthermore, if $\varphi_{1}, \varphi_{2}$ are disjoint states on $\mathfrak{A}_{E}$, the traces $\varphi_{1 \omega}$ and $\varphi_{2 \omega}$ are disjoint on $\mathfrak{A}_{E \omega}$. To see this, let $F$ be the central support for $\varphi_{1}$ in $\mathfrak{A}_{E}^{* *}$, and pick a sequence $x_{n} \in \mathfrak{A}_{E}$ such that $\left\|x_{n}\right\| \leq 1$ and $s-\lim _{n \rightarrow \omega} x_{n}=F$. Since $F$ is central, we have $U F U^{*}=F$ for all $U \in \mathcal{U}\left(\mathfrak{A}_{m E}\right)$, and by approximating the projection $\int_{\mathcal{U}\left(\mathfrak{A}_{m E}\right)} U \cdot U^{*} d U$ onto $\mathfrak{A}_{m E}^{\prime}$ by finite convex combinations, we may replace $x_{n}$ by a sequence such that there is a sequence $k_{n} \in \mathbf{N}$ such that $\left(k_{n}\right)$ is increasing, $k_{n} \rightarrow \infty$ and $x_{n} \in E\left(\mathfrak{A} \cap \mathfrak{A}_{k_{n}}^{\prime}\right) E$ for all $n$. Then the new sequence $x=\left(x_{n}\right)$ is norm central and corresponds to an element $\widetilde{F}$ in the unit ball of $\mathfrak{A}_{E \omega}$. But then $\varphi_{1 \omega}(\widetilde{F})=1, \varphi_{2 \omega}(\widetilde{F})=0$, so the trace states $\varphi_{1 \omega}$ and $\varphi_{2 \omega}$ are disjoint. We have defined the tracial states $\tau_{1 \omega}, \ldots, \tau_{d \omega}$ of $\mathfrak{A}_{E \omega}$ and shown that they are mutually disjoint. Let $\pi_{i \omega}$ be the GNS representation of $\mathfrak{A}_{E \omega}$ associated to $\tau_{i \omega}$ and consider the sum $\pi_{\omega}=\bigoplus_{i=1}^{d} \pi_{i \omega}$. If $\mathfrak{R}=\pi_{\omega}\left(\mathfrak{A}_{E \omega}\right)^{\prime \prime}=\bigoplus_{i=1}^{d} \pi_{i \omega}\left(\mathfrak{A}_{E \omega}\right)^{\prime \prime}, \widetilde{\alpha}_{\omega}$ extends to an automorphism of $\mathfrak{R}$, which is denoted again by $\widetilde{\alpha}_{\omega}^{i=1}$.

Lemma 2.4. For any $n \in \mathbf{Z} \backslash\{0\}, \widetilde{\alpha}_{\omega}^{n}$ is a properly outer automorphism of $\mathfrak{R}$.

Proof. Suppose that $\pi_{i \omega} \circ \widetilde{\alpha}_{\omega}^{n}=\pi_{i \omega}$. Then as in the proof of Lemma 2.4 of [EK] we argue that $\widetilde{\alpha}_{\omega}^{n} \mid \pi_{i \omega}\left(\mathfrak{A}_{E \omega}\right)^{\prime \prime}$ is properly outer (where we use the fact that $\pi_{i \omega}\left(\mathfrak{A}_{E \omega}\right)^{\prime \prime}=$ $\pi_{i \omega}\left(\mathfrak{A}_{E \omega}\right)=\mathfrak{R}_{i \omega}$ for $\left.\mathfrak{R}_{i}=\pi_{i}\left(\mathfrak{A}_{E}\right)^{\prime \prime}\right)$. Thus we conclude that $\widetilde{\alpha}_{\omega}^{n}$ is properly outer on $\mathfrak{R}$.

Lemma 2.5. Recall that $k_{1}, k_{2}-k_{1}, k_{3}-k_{2}, \cdots, k_{l}-k_{l-1}$ are the periods for the cycles in $\left\{\tau_{1 \omega}, \ldots, \tau_{d \omega}\right\}$ under the action of $\widetilde{\alpha}_{\omega}$. Let again $p$ be the least common multiple of $k_{1}, k_{2}-k_{1}, \ldots, k_{l}-k_{l-1}$. Then for any $n \in \mathbf{N}$ there are np projections $F_{0}, F_{1}, \ldots, F_{n p-1}$ in $\mathfrak{R}$ such that

$$
\begin{aligned}
& \sum_{i=0}^{n p-1} F_{i}=\mathbb{1}, \\
& \widetilde{\alpha}_{\omega}\left(F_{i}\right)=F_{i+1}, \quad i=0,1, \ldots, n p-1 \\
& \tau_{k \omega}\left(F_{i}\right)=\frac{1}{n p}, \quad k=1,2, \ldots, d
\end{aligned}
$$


where $F_{n p}=F_{0}$.

Proof. Without the condition $\tau_{k \omega}\left(F_{i}\right)=\frac{1}{n p}$, this follows from C75.

To obtain this condition we consider each cycle $\left\{\tau_{k_{i+1} \omega}, \ldots, \tau_{k_{i+1}}\right\}$ separately, and thus we may suppose that $\left\{\tau_{1 \omega}, \ldots, \tau_{\alpha \omega}\right\}$ is one cycle, i.e., $\tau_{i \omega} \circ \widetilde{\alpha}=\tau_{i+1 \omega}$ except $\tau_{d \omega} \circ \widetilde{\alpha}_{\omega}=\tau_{1 \omega}$. In this case we find $n d$ projections $F_{i}^{(j)}, j=1,2, \ldots, d$, $i=0,1, \ldots, n-1$ in $\pi_{1 \omega}\left(\mathfrak{A}_{E \omega}\right)^{\prime \prime}$ such that

$$
\begin{aligned}
& \sum_{j=1}^{d} \sum_{i=0}^{n-1} F_{i}^{(j)}=\mathbb{1} \quad \text { in } \pi_{1 \omega}\left(\mathfrak{A}_{E \omega}\right)^{\prime \prime}, \\
& \widetilde{\alpha}_{\omega}^{d}\left(F_{i}^{(j)}\right)=F_{i+1}^{(j)}, \quad i=0,1, \ldots, n-1
\end{aligned}
$$

where $F_{n}^{(j)}=F_{0}^{(j)}$. Then we set

$$
F_{i}=\sum_{j=1}^{d} \widetilde{\alpha}_{\omega}^{i+j-1}\left(F_{0}^{(j)}\right)
$$

for $i=0,1, \ldots, n d-1$. It follows that

$$
\begin{aligned}
& \sum_{i=0}^{n d-1} F_{i}=\mathbb{1} \quad \text { in } \mathfrak{R}, \\
& \widetilde{\alpha}_{\omega}\left(F_{i}\right)=F_{i+1}, \\
& \tau_{k \omega}\left(F_{i}\right)=\frac{1}{n d} .
\end{aligned}
$$

Lemma 2.6. There exists an orthogonal family $\left\{f_{0}, f_{1}, \ldots, f_{n p-1}\right\}$ of projections in $\mathfrak{A}_{E \omega}$ such that

$$
\begin{aligned}
& \widetilde{\alpha}_{\omega}\left(f_{i}\right)=f_{i+1} \quad i=0,1, \ldots, n p-2 . \\
& \tau_{k \omega}\left(f_{i}\right)=\frac{1}{n p} .
\end{aligned}
$$

Proof. For each $F_{i}$ in Lemma 2.5 we find a projection $f_{i} \in \mathfrak{A}_{E \omega}$ such that $F_{i}=$ $\pi_{\omega}\left(f_{i}\right)$. However this only shows that $\pi_{\omega}\left(\widetilde{\alpha}_{\omega}\left(f_{i}\right)\right)=\pi_{\omega}\left(f_{i+1}\right)$, and $\pi_{\omega}$ will never be faithful. To overcome this difficulty we replace $f_{0}$ by a smaller projection and we set $f_{i}=\widetilde{\alpha}_{\omega}^{i}\left(f_{0}\right)$ as in [K95, K96]. Note that we have not assumed that $\widetilde{\alpha}_{\omega}\left(f_{n p-1}\right)=$ $f_{0}$.

Lemma 2.7. Recall the overall assumptiuon in Theorem 2.1 that the image of $\mathbf{Z}\left[x, x^{-1}\right]$ in $C(\Lambda)=C\left(\left\{\lambda_{1}^{p}, \ldots, \lambda_{l}^{p}\right\}\right)$ is dense. There exists a sequence $\left(f_{0 m}\right)$ of projections in $\mathfrak{A}_{E}$ such that $f_{0 m} \in\left(\mathfrak{A}_{E} \cap \mathfrak{A}_{k_{m}}^{\prime}\right)_{E}$ with $k_{m} \rightarrow \infty$ as $m \rightarrow \infty,\left(f_{0 m}\right)$ in $\mathfrak{A}_{E \omega}$ is less than $f_{0}$ in Lemma 2.6,

$$
\tau_{k \omega}\left(\left(f_{0 m}\right)\right)=\frac{1}{n p} \quad \text { for } k=1,2, \ldots, d
$$

and

$$
\left[f_{0 m} p_{j}^{\left(k_{m}\right)}\right]=p_{m}\left(\alpha_{*}^{p}, \alpha_{*}^{-p}\right)\left[p_{j}^{\left(k_{m}\right)}\right],
$$

in $K_{0}\left(\left(\mathfrak{A} \cap \mathfrak{A}_{k_{m}}^{\prime}\right)_{E}\right)$ for some $p_{m} \in \mathbf{Z}\left[x, x^{-1}\right]$, where $p_{j}^{\left(k_{m}\right)}$ denotes $p_{j}^{\left(k_{m}\right)} E$. 
Proof. First we take a sequence $\left(f_{0 m}\right)$ of projections in $\mathfrak{A}_{E}$ which represents $f_{0} \in$ $\mathfrak{A}_{E \omega}$ in Lemma 2.6 and satisfies that $f_{0 m} \in\left(\mathfrak{A} \cap \mathfrak{A}_{k_{m}}^{\prime}\right)_{E}$ and for $p_{j}^{(k)}$ we have that

$$
\tau_{s}\left(f_{0 m} p_{j}^{(k)}\right) \rightarrow \frac{1}{n p} \tau_{s}\left(p_{j}^{(k)}\right) \quad \text { as } m \rightarrow \infty .
$$

For any $\varepsilon>0$ with $\varepsilon<\frac{1}{2 n p}$ it follows from the density of $\mathbf{Z}\left[x, x^{-1}\right]$ in $C(\Lambda)$ that there exists a function $q \in \mathbf{Z}\left[x, x^{-1}\right]$ such that

$$
\frac{1}{n p}-2 \varepsilon<q\left(\lambda_{i}^{p}, \lambda_{i}^{-p}\right)<\frac{1}{n p}-\varepsilon .
$$

If $k_{i-1}<s \leq k_{i}$ then

$$
\tau_{s}\left(q\left(\alpha^{p}, \alpha^{-p}\right) p_{j}^{(n)}\right)=q\left(\lambda_{i}^{p}, \lambda_{i}^{-p}\right) \tau_{s}\left(p_{j}^{(n)}\right)
$$

since $\tau_{s} \alpha^{p}=\lambda_{i}^{p} \tau_{s}$. Thus for a sufficiently large $m$, it follows that

$$
q\left(\alpha_{*}^{p}, \alpha_{*}^{-p}\right)\left[p_{j}^{(n)}\right] \leq\left[f_{0 m} p_{j}^{(n)}\right]
$$

in $K_{0}\left(\left(\mathfrak{A} \cap \mathfrak{A}_{k}^{\prime}\right)_{E}\right.$. Then we find a subprojection $f_{0 m}^{\prime}$ of $f_{0 m}$ in $\left(\mathfrak{A} \cap \mathfrak{A}_{n}^{\prime}\right)_{E}$ such that

$$
\left[f_{0 m}^{\prime} p_{j}^{(n)}\right]=q\left(\alpha_{*}^{p}, \alpha_{*}^{-p}\right)\left[p_{j}^{(n)}\right] .
$$

For an increasing $k$ and a decreasing $\varepsilon$ we use the above argument to replace $f_{0 m}$ by a smaller projection satisfying the required properties.

Proof of Theorem 1.2. By using the projections $f_{0 m}$ in Lemma 2.7 we obtain that

$$
f_{0 m}, \widetilde{\alpha}\left(f_{0 m}\right), \widetilde{\alpha}^{2}\left(f_{0 m}\right), \ldots, \widetilde{\alpha}^{n p-1}\left(f_{0 m}\right)
$$

are almost mutually orthogonal when $m \rightarrow \omega$ and we must show that they are equivalent as projections in $\left(\mathfrak{A} \cap \mathfrak{A}_{k_{m}-n p}^{\prime}\right)_{E}$.

To show that they are equivalent, we regard $\widetilde{\alpha}, \iota$ as homomorphisms of $\left(\mathfrak{A} \cap \mathfrak{A}_{k_{m}}^{\prime}\right)_{E}$ into $\left(\mathfrak{A} \cap \mathfrak{A}_{k_{m}-1}^{\prime}\right)_{E}$ and compute by Lemmas $2.1-2.3$

$$
\begin{aligned}
\widetilde{\alpha}_{*}\left(\left[f_{0 m}\right]\right) & =\left(\sum_{j} \widetilde{\alpha}_{*}(i, j)\left(\left[f_{0 m} p_{j}^{\left(k_{m}\right)}\right]\right)\right)_{i} \\
& =\left(\sum_{j} \widetilde{\alpha}_{*}(i, j)\left(p_{m}\left(\alpha_{*}^{p}, \alpha_{*}^{-p}\right)\left[p_{j}^{\left(k_{m}\right)}\right]\right)\right)_{i} \\
& \left.=\left(p_{m}\left(\alpha_{*}^{p}, \alpha_{*}^{-p}\right) \sum_{j} \widetilde{\alpha}_{*}(i, j)\left[p_{j}^{\left(k_{m}\right)}\right]\right)\right)_{i} \\
& =\left(p_{m}\left(\alpha_{*}^{p}, \alpha_{*}^{-p}\right)\left[p_{i}^{\left(k_{m}-1\right)}\right]\right)_{i} .
\end{aligned}
$$

With a similar computation for $\iota_{*}$ we obtain that

$$
\begin{aligned}
{\left[\widetilde{\alpha}\left(f_{0 m}\right)\right] } & =\widetilde{\alpha}_{*}\left(\left[f_{0 m}\right]\right) \\
& =\left(p_{m}\left(\alpha_{*}^{p}, \alpha_{*}^{-p}\right)\left[p_{i}^{\left(k_{m}-1\right)}\right]\right)_{i} \\
& =\iota_{*}\left(\left[f_{0 m}\right]\right),
\end{aligned}
$$

i.e., $\left[\widetilde{\alpha}\left(f_{0 m}\right)\right]=\left[f_{0 m}\right]$ in $K_{0}\left(\left(\mathfrak{A} \cap \mathfrak{A}_{k_{m}-1}^{\prime}\right)_{E}\right)$. We can repeat this process. Thus we find a $\mathrm{C}^{*}$-subalgebra $\mathfrak{B}$ of $\left(\mathfrak{A} \cap \mathfrak{A}_{k_{m}-n p}^{\prime}\right)_{E}$ such that $\mathfrak{B}$ is a factor of type $I_{n p}$ which almost contains the $n p$ projections $f_{0 m}, \widetilde{\alpha}\left(f_{0 m}\right), \ldots, \widetilde{\alpha}^{n p-1}\left(f_{0 m}\right)$, the identity of $\mathfrak{B}$ is close to $E$ when the distance is measured with the traces $\tau_{s}$, and the large portion of $\mathfrak{B}$ is $\widetilde{\alpha}$-invariant. ¿From this one can deduce the Rohlin property for $\widetilde{\alpha}$ as in 
K95, K96]. By reinterpreting $\widetilde{\alpha}$ in terms of $\operatorname{Ad} U \circ \alpha$ and $e$, one obtains the Rohlin property of $\alpha$.

After having finished the proof of Theorem 1.2 we now consider the special condition on the ring $\mathbf{Z}\left[x, x^{-1}\right]$ of Laurent polynomials, i.e. we establish the statements in Remark 1.3.

Proposition 2.8. Let $\Lambda$ be a compact subset of $(0,1) \cup(1, \infty)$. Then $\mathbf{Z}\left[x, x^{-1}\right]$ is dense in $C(\Lambda)$ if one of the following conditions hold:

1. $\Lambda \subset C(0,2)$.

2. $\Lambda \subset(1 / 2, \infty)$.

3. $\Lambda$ is a finite subset of the rational numbers $\mathbf{Q}$.

Proof. By Corollary 9.3 of $\left[\mathrm{Fer}\right.$ it suffices to show that there is a $p \in \mathbf{Z}\left[x, x^{-1}\right]$ such that $\|p\|_{\Lambda}<1$ and $p\left(\lambda, \lambda^{-1}\right) \neq 0$ for $x \in \Lambda$.

(The existence of $p$ is clearly necessary for the density. For the sufficiency, let us extract the apposite argument from [Fer]: Let $f \in C(\Lambda)$ and put $g=f / p$. Then $g \in C(\Lambda)$. By Weierstrass theorem, there exists a real polynomial $q(x)=\sum_{k=0}^{r} \beta_{k} x^{k}$ approximating $g$, and we may assume that each $\beta_{k}$ has the form $\beta=n 2^{-m}$. If $a=\inf p>0$ and $b=\sup p<1$ we may approximate each $\beta$ arbitrarily well on $[a, b]$ by

$$
n \frac{1}{\left(2-t^{k}\right)^{m}}=\frac{n}{\left(1-\left(t^{k}-1\right)\right)^{m}}=n\left(\sum_{i=1}^{\infty}\left(t^{k}-1\right)^{i}\right)^{m}
$$

by choosing $k$ large and replacing $\infty$ by a finite number, and thus we may approximate the first order polynomials $\beta_{k} t$ arbitrarily well by polynomials $p_{k}(t) \in \mathbf{Z}[t]$ for $t \in[a, b]$. Thus

$$
q(x) p(x)-\sum_{k=0}^{r} x^{k} p_{k}(p(x))=\sum_{k=0}^{r} x^{k}\left(\beta_{k} p(x)-p_{k}(p(x))\right)
$$

is uniformly small for $x \in \Lambda$, and since $f(x)=g(x) p(x) \approx q(x) p(x)$ and $\sum_{k=0}^{r} x^{k} p^{k}(p(x)) \in$ $\mathbf{Z}\left[x, x^{-1}\right]$, the sufficiency follows.)

We may use $p=x(2-x)$ to establish (1) and $p=\frac{1}{x}\left(2-\frac{1}{x}\right)$ to establish (2) in the Proposition.

In case (3), let $\Lambda \cap(0,1)=\left\{\frac{p_{i}}{q_{i}}: i=1, \ldots, k\right\}$ and $\Lambda \cap(1, \infty)=\left\{\frac{p_{i}}{q_{i}}: i=\right.$ $k+1, \ldots, \ell\}$, where $p_{i}, q_{i}$ are integers, and let

$$
P\left(x, x^{-1}\right)=\frac{1}{x^{n}} \prod_{i=1}^{k}\left(q_{i} x-p_{i}\right)^{2}+x^{n} \prod_{i=k+1}^{\ell}\left(q_{i} x-p_{i}\right)^{2}
$$

for a sufficiently large $n$.

Example 2.9. If $\Lambda=\{2-\sqrt{3}, 2+\sqrt{3}\}$ the image of $\mathbf{Z}\left[x, x^{-1}\right]$ in $C(\Lambda)$ is not dense.

Note that $\lambda_{1}=2-\sqrt{3}$ and $\lambda_{2}=\frac{1}{\lambda_{1}}=2+\sqrt{3}$ are the roots of the equations

$$
x^{2}-4 x+1=0
$$

and

$$
\frac{1}{x^{2}}-\frac{4}{x}+1=0
$$


Thus it suffices to show that the image of

$$
\left\{a x+\frac{b}{x}+c \mid a, b, c \in \mathbf{Z}\right\}
$$

in $C\left(\left\{\lambda_{1}, \lambda_{2}\right\}\right)$ is not dense, or

$$
\left\{\left(a \lambda_{1}+b \lambda_{2}+c, a \lambda_{2}+b \lambda_{1}+c\right) \mid a, b, c \in \mathbf{Z}\right\}
$$

is not dense in $\mathbf{R}^{2}$. Suppose that for some $a, b, c \in \mathbf{Z}$.

$$
0<a \lambda_{1}+b \lambda_{2}+c<1, \quad 0<a \lambda_{2}+b \lambda_{1}+c<1 .
$$

If $a=b$, then $a \lambda_{1}+b \lambda_{2}+c=4 a+c \in \mathbf{Z}$, which is a contradiction. Hence $a \neq b$. Since

$$
(a-b) \lambda_{1}-(a-b) \lambda_{2}=2(a-b) \sqrt{3}
$$

has modulus at least 1 , we reach a contradition. Thus the assertion follows.

Example 2.10. In this example we show how to construct $\mathrm{C}^{*}$-dynamical systems $(\mathfrak{A}, \alpha)$ satisfying the hypotheses in Theorem 1.2 . Let $\Lambda$ be a finite subset of $(0,1) \cup$ $(1, \infty)$ and let $d \in \mathbf{N}$. We define an order on $\bigoplus_{1}^{d} \mathbf{Z}\left[x, x^{-1}\right]$ by $p \geq q$ if $p_{i}(\lambda)>q_{i}(\lambda)$ for $i=1,2, \ldots, d$ and $\lambda \in \Lambda$ or $p_{i} \equiv q_{i}$ for $i=1,2, \ldots, d$ where $p=\left(p_{1}, p_{2}, \ldots, p_{d}\right)$ etc. If the image of $\mathbf{Z}\left[x, x^{-1}\right]$ in $C(\Lambda)$ is dense, then this order on $\bigoplus_{1}^{d} \mathbf{Z}\left[x, x^{-1}\right]$ gives a dimension group, which will be denoted by $G$. We define an automorphism $\alpha_{*}$ of $G$ by

$$
\alpha_{*}\left(\left(p_{1}, p_{2} \ldots, p_{d}\right)\right)=\left(x p_{d}, p_{1}, p_{2}, \ldots, p_{d-1}\right) .
$$

$G$ is associated to a stable AF algebra $\mathfrak{A}$ and $\alpha_{*}$ to an automorphism $\alpha$ of $\mathfrak{A}$. For each $i=1,2, \ldots, d$ and $\lambda \in \Lambda$ there is a positive character $\tau_{i, \lambda}$ on $G$ such that $\tau_{i, \lambda}(p)=p_{i}(\lambda)$ for $p=\left(p_{1}, p_{2}, \ldots, p_{d}\right) \in G$. Each $\tau_{i, \lambda}$ gives a lower semicontinuous trace on $\mathfrak{A}$, which we denote by the same symbol. Since

$$
\tau_{i, \lambda} \alpha= \begin{cases}\tau_{i-1, \lambda} & \text { for } i=2,3, \ldots, d \\ \lambda \tau_{d, \lambda} & \text { for } i=1\end{cases}
$$

it follows that $\tau_{i \lambda} \alpha^{d}=\lambda \tau_{i \lambda}$ for all $i, \lambda$ and thus the set of scales is $\Lambda$. Since $(\mathfrak{A}, \alpha)$ satisfies the assumptions in Theorem 1.2, $\alpha$ has the Rohlin property. In passing we can see that

$$
G /\left(\mathrm{id}-\alpha_{*}\right) G \cong \mathbf{Z} .
$$

Since $\mathfrak{A} \times_{\alpha} \mathbf{Z}$ is purely infinite [R1], $\mathfrak{A} \times_{\alpha} \mathbf{Z}$ must be isomorphic to $O_{\infty} \otimes \mathfrak{K}[\mathrm{KP}$. (This conclusion also holds if $\Lambda$ is a non-finite closed subset of $(0,1) \cup(1, \infty)$ such that the dimension group condition is satisfied, as it is when $\bigoplus_{1}^{d} \mathbf{Z}\left[x, x^{-1}\right]$ is dense in $C(\Lambda)$.)

Remark 2.11. When there is an automorphism $\beta$ of $K_{0}(\mathfrak{A})$ such that $\beta \alpha_{*}=\alpha_{*} \beta$, we obtain that $\widetilde{\alpha}_{*}(i, j) \beta=\beta \widetilde{\alpha}_{*}(i, j)$ and $\iota_{*}(i, j) \beta=\beta \iota_{*}(i, j)$ as in Lemmas 2.1 and 2.2 and we can sometimes use this fact in Lemma 2.7 to prove that $\alpha$ has the Rohlin property. As an example, take a $\lambda \in(0,1 / 2)$ and equip the abelian group $\mathbf{Z}\left[x, x^{-1},(1-x)^{-1}\right]$ with the order defined by $p \geq 0$ if $p\left(\lambda, \lambda^{-1},(1-\lambda)^{-1}\right)>0$ and $p\left(\frac{1}{2}, 2,2\right)>0$, or $p \equiv 0$. Let $\mathfrak{A}$ be the stable AF algebra corresponding to this ordered group and $\alpha$ an automorphism of $\mathfrak{A}$ corresponding to the multiplication of $\frac{1-x}{x}$ such that in the tracial representation corresponding to $1 / 2$ all non-zero 
poweres of $\alpha$ are not weakly inner. (For the tracial representation corresponding to $\lambda$ this follows automatically.) The automorphism $\beta$ of $K_{0}(\mathfrak{A})$ corresponding to multiplication by $x$ commutes with $\alpha_{*}$. To prove that $\alpha$ has the Rohlin property we proceed exactly as before but use polynomials in $\beta$ and $\beta^{-1}$ in Lemma 2.7. (Since $\beta$ scales the traces by $1 / 2$ and $\lambda$, the required condition for 2.7 is satisfied; the image of $\mathbf{Z}\left[x, x^{-1}\right]$ in $C(\{\lambda, 1 / 2\})$ is dense. In passing we note that the quotient of $K_{0}(\mathfrak{A})$ by $\operatorname{Im}\left(\mathrm{id}-\alpha_{*}\right)$ is isomorphic to $\mathbf{Z}\left[\frac{1}{2}\right]$, the dimension group of the UHF algebra of type $2^{\infty}$. Since $\operatorname{ker}\left(\mathrm{id}-\alpha_{*}\right)=0$, it follows that $\mathfrak{A} \times_{\alpha} \mathbf{Z}$ has the same $K$-theory as the UHF algebra of type $2^{\infty}$. (The automorphism $\alpha$ was first used in [BEH].)

Remark 2.12. By taking the tensor product of $\mathfrak{A}$ with a UHF algebra $\mathfrak{B}$, we could overcome the difficulty encountered in Lemma 2.7. We could thus show in Theorem 1.2 without the condition on the scaling factors that $\alpha \otimes$ id on $\mathfrak{A} \otimes \mathfrak{B}$ has the Rohlin property.

\section{Actions of a compact abelian group}

We will describe here some invariants for conjugacy and cocycle conjugacy classes of actions of a compact abelian group on a unital $\mathrm{C}^{*}$-algebra, borrowing ideas from Bla], HR.

Let $\mathfrak{A}$ be a unital $\mathrm{C}^{*}$-algebra and $\alpha$ an action of a compact abelian group $G$ on $\mathfrak{A}$. The dual system is $\left(\mathfrak{A} \times{ }_{\alpha} G, \widehat{G}, \widehat{\alpha}\right)$, where $\widehat{G}$ is the dual group of $G$. The dual system of $\left(\mathfrak{A} \times{ }_{\alpha} G, \widehat{G}, \widehat{\alpha}\right)$ is isomorphic to $\left(\mathfrak{A} \otimes \mathfrak{K}\left(L^{2}(G)\right), G, \alpha \otimes \operatorname{Ad} \widetilde{\lambda}\right)$ by Takai's duality Tak, where $\mathfrak{K}\left(L^{2}(G)\right)$ is the compact operators on $L^{2}(G)$ and $\widetilde{\lambda}$ is the unitary representation of $G$ on $L^{2}(G)$ defined by

$$
\left(\widetilde{\lambda}_{t} \xi\right)(s)=\xi(t+s)
$$

¿From the inclusion $\iota: \mathfrak{A} \times{ }_{\alpha} G \subset \mathfrak{A} \otimes \mathfrak{K}\left(L^{2}(G)\right)$ we obtain the natural map $\iota_{*}$ : $K_{i}\left(\mathfrak{A} \times_{\alpha} G\right) \rightarrow K_{i}(\mathfrak{A})$ for $i=0,1$ which satisfies that $\iota_{*} \widehat{\alpha}(s)_{*}=\iota_{*}, s \in \widehat{G}$.

Let $\lambda$ be the canonical unitary representation of $G$ in the multiplier algebra $M\left(\mathfrak{A} \times{ }_{\alpha} G\right)$ of $\mathfrak{A} \times{ }_{\alpha} G$. Let $\left\{P_{\alpha}(s) ; s \in \widehat{G}\right\}$ be the spectral projections for $\lambda$ :

$$
\lambda_{t}=\sum_{s \in \widehat{G}}\langle t, s\rangle P_{\alpha}(s)
$$

Then it follows that $\iota_{*}\left(\left[P_{\alpha}(0)\right]\right)=[\mathbb{1}]$, where $\mathbb{1}$ is the identity element of $\mathfrak{A}$. Thus to each action $\alpha$ of $G$ on $\mathfrak{A}$ we associate the following $K$-theoretic data:

$$
\begin{array}{rll}
K_{0}\left(\mathfrak{A} \times_{\alpha} G\right) & \stackrel{\iota_{*}}{\longrightarrow} K_{0}(\mathfrak{A}) \\
{\left[P_{\alpha}(0)\right]} & \mapsto & {[\mathbb{1}]} \\
K_{1}\left(\mathfrak{A} \times_{\alpha} G\right) & \stackrel{\iota_{*}}{\longrightarrow} K_{1}(\mathfrak{A})
\end{array}
$$

with the action $\widehat{\alpha}_{*}$ of $\widehat{G}$ on $K_{i}\left(\mathfrak{A} \times{ }_{\alpha} G\right)$ satisfying $\iota_{*} \widehat{\alpha}_{*}(s)=\iota_{*}$.

Proposition 3.1. Let $\mathfrak{A}$ be a unital $C^{*}$-algebra and let $\alpha, \beta$ be actions of a compact abelian group $G$ on $\mathfrak{A}$. If $\alpha$ is conjugate to $\beta$, then there exist isomorphisms $\varphi_{1}$ of $K_{i}\left(\mathfrak{A} \times_{\alpha} G\right)$ onto $K_{i}\left(\mathfrak{A} \times_{\beta} G\right)$ and $\varphi_{2}$ of $K_{i}(\mathfrak{A})$ onto $K_{i}(\mathfrak{A})$ such that

$$
\begin{aligned}
& \iota_{*} \varphi_{1}=\varphi_{2} \iota_{*} \\
& \varphi_{1} \widehat{\alpha}_{*}(s)=\widehat{\beta}_{*}(s) \varphi_{1} \\
& \varphi_{1}\left(\left[P_{\alpha}(0)\right]\right)=\left[P_{\beta}(0)\right]
\end{aligned}
$$

where $\varphi_{1}, \varphi_{2}$ are order isomorphisms if applicable. 
Proof. Note that $\varphi_{2}([\mathbb{1}])=[\mathbb{1}]$ follows automatically.

If $\alpha=\sigma \beta \sigma^{-1}$ for an automorphism $\sigma$ of $\mathfrak{A}$, then there is an isomorphism $\phi$ of $\mathfrak{A} \times{ }_{\alpha} G$ onto $\mathfrak{A} \times{ }_{\beta} G$ such that

$$
\begin{array}{ll}
\phi(a)=\sigma^{-1}(a), & a \in \mathfrak{A} \\
\phi(\lambda(t))=\lambda(t), & t \in G
\end{array}
$$

By setting $\varphi_{1}=\phi_{*}$ and $\varphi_{2}=\sigma_{*}^{-1}$, all the properties follow easily.

Proposition 3.2. Let $\mathfrak{A}$ be a unital $C^{*}$-algebra and let $\alpha, \beta$ be actions of a compact abelian group $G$ on $\mathfrak{A}$. If $\alpha$ is cocycle conjugate to $\beta$, then there exist isomorphisms $\varphi_{1}$ of $K_{i}\left(\mathfrak{A} \times{ }_{\alpha} G\right)$ onto $K_{i}\left(\mathfrak{A} \times_{\beta} G\right)$ and $\varphi_{2}$ of $K_{i}(\mathfrak{A})$ onto $K_{i}(\mathfrak{A})$ such that

$$
\begin{aligned}
& \iota_{*} \varphi_{1}=\varphi_{2} \iota_{*} \\
& \varphi_{1} \widehat{\alpha}_{*}(s)=\widehat{\beta}_{*}(s) \varphi_{1} \\
& \varphi_{2}([1])=[1]
\end{aligned}
$$

where $\varphi_{1}, \varphi_{2}$ are order isomorphisms if applicable.

Proof. In view of Proposition 3.1 it suffices to show this when $\alpha$ is a cocycle perturbation of $\beta$, i.e., $\alpha_{t}=\operatorname{Ad} u_{t} \circ \beta_{t}$ with $u_{t}$ a one-cocycle for $\beta$.

We define an isomorphism $\phi$ of $\mathfrak{A} \times{ }_{\alpha} G$ onto $\mathfrak{A} \times{ }_{\beta} G$ by

$$
\begin{aligned}
& \phi(a)=a, \quad a \in \mathfrak{A} \\
& \phi(\lambda(t))=u_{t} \lambda(t), \quad t \in G
\end{aligned}
$$

Since $\widehat{\beta}(s) \phi=\phi \widehat{\alpha}(s), s \in \widehat{G}, \phi$ naturally extends to an isomorphism $\widehat{\phi}$ of $\mathfrak{A} \times{ }_{\alpha} G \times{ }_{\widehat{\alpha}} \widehat{G}$ onto $\mathfrak{A} \times{ }_{\beta} G \times{ }_{\widehat{\beta}} \widehat{G}$. By setting $\varphi_{1}=\phi_{*}$ and $\varphi_{2}=\widehat{\phi}_{*}$, all the properties follow easily, perhaps except for $\varphi_{2}([\mathbb{1}])=[\mathbb{1}]$. For this we shall show that $\varphi_{2}=$ id.

Supposing that $\mathfrak{A}$ is represented on a Hilbert space $\mathcal{H}$, we represent $\mathfrak{A} \times{ }_{\alpha} G \times_{\widehat{\alpha}} G$ on $L^{2}(G, \mathcal{H})$ by

$$
\begin{aligned}
& \left(\pi_{\alpha}(a) \xi\right)(s)=\alpha_{-s}(a) \xi(s) \\
& (\lambda(t) \xi)(s)=\xi(s-t) \\
& (v(p) \xi)(s)=\langle p, s\rangle \xi(s)
\end{aligned}
$$

for $a \in \mathfrak{A}, s, t \in G$, and $p \in \widehat{G}$. Then $\mathfrak{A} \times_{\alpha} G$ is generated by $\pi_{\alpha}(\mathfrak{A})$ and $\lambda(f)$, $f \in L^{1}(G)$ and $\mathfrak{A} \times{ }_{\alpha} G \times \times_{\widehat{\alpha}} \widehat{G}$ is generated by $\left(\mathfrak{A} \times{ }_{\alpha} G\right) v(f), f \in L^{1}(\widehat{G})$, which naturally identifies with $\mathfrak{A} \otimes \mathfrak{K}\left(L^{2}(G)\right)$. Then $\widehat{\phi}$ is given by

$$
\begin{aligned}
& \pi_{\alpha}(a) \mapsto \pi_{\beta}(a) \\
& \lambda(t) \mapsto u_{t} \lambda(t) \\
& v(p) \mapsto v(p)
\end{aligned}
$$

and $\widehat{\phi}$ is implemented by the unitary $U$ defined by

$$
(U \xi)(s)=u_{-s}^{*} \xi(s),
$$

which is a multiplier of $\mathfrak{A} \otimes \mathfrak{K}\left(L^{2}(G)\right)$. Thus we obtain the commutative diagram:

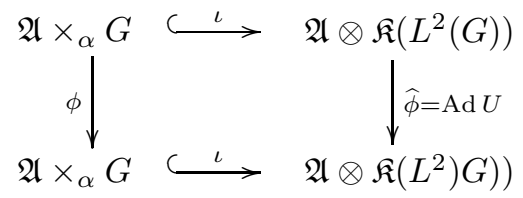

Thus the assertion follows. 


\section{Conjugacy and Cocycle Conjugacy Classes of aCtions of T}

As an application of Theorem 1.2 we consider the problem of classifying a class of actions of $\mathbf{T}$ on a unital separable simple purely infinite $\mathrm{C}^{*}$-algebra $\mathfrak{A}$.

To meet the assumption in Theorem 1.2 we have to assume that $\mathfrak{A} \times_{\alpha} \mathbf{T}$ is AF, which implies in particular that $\mathfrak{A}$ is nuclear. By the Pimsner-Voiculescu exact sequence, $\iota_{*}: K_{0}\left(\mathfrak{A} \times_{\alpha} \mathbf{T}\right) \rightarrow K_{0}(\mathfrak{A})$ is just the quotient map obtained by division by $\operatorname{Im}\left(\mathrm{id}-\widehat{\alpha}_{*}\right)$, where we now denote by $\widehat{\alpha}$ the single automorphism $\widehat{\alpha}(1)$ of $\mathfrak{A} \times_{\alpha} \mathbf{T}$. Thus the invariants for conjugacy classes described in 3.1 reduce to

$$
\left(K_{0}\left(\mathfrak{A} \times_{\alpha} \mathbf{T}\right),\left[P_{\alpha}(0)\right], \widehat{\alpha}_{*}\right),
$$

and the following relations hold in this case:

$$
\begin{aligned}
& \left(K_{0}\left(\mathfrak{A} \times_{\alpha} \mathbf{T}\right) / \operatorname{Im}\left(\mathrm{id}-\widehat{\alpha}_{*}\right), \quad\left[P_{\alpha}(0)\right]+\operatorname{Im}\left(\mathrm{id}-\widehat{\alpha}_{*}\right)\right) \cong\left(K_{0}(\mathfrak{A}),[1]\right) \\
& \operatorname{ker}\left(\left(\mathrm{id}-\widehat{\alpha}_{*}\right) \mid K_{0}\left(\mathfrak{A} \times_{\alpha} \mathbf{T}\right) \cong K_{1}(\mathfrak{A}) .\right.
\end{aligned}
$$

Corollary 4.1. Let $\mathfrak{A}$ be a unital separable simple purely infinite $C^{*}$-algebra and let $\alpha, \beta$ be actions of $\mathbf{T}$ on $\mathfrak{A}$ such that $\mathfrak{A} \times{ }_{\alpha} \mathbf{T}$ and $\mathfrak{A} \times{ }_{\beta} \mathbf{T}$ are simple AF algebras with one-dimensional lattices of traces. Then the following conditions are equivalent:

1. $\alpha$ and $\beta$ are conjugate

2. $\left(K_{0}\left(\mathfrak{A} \times_{\alpha} \mathbf{T}\right),\left[P_{\alpha}(0)\right], \widehat{\alpha}_{*}\right)$ and $\left(K_{0}\left(\mathfrak{A} \times_{\beta} \mathbf{T}\right),\left[P_{\beta}(0)\right], \widehat{\beta}_{*}\right)$ are isomorphic, i.e., there is an order-isomorphism $\varphi_{1}$ of $K_{0}\left(\mathfrak{A} \times_{\alpha} \mathbf{T}\right)$ onto $K_{0}\left(\mathfrak{A} \times_{\beta} \mathbf{T}\right)$ such that $\varphi_{1} \widehat{\alpha}_{*}=\widehat{\beta}_{*} \varphi_{1}$ and $\varphi_{1}\left(\left[P_{\alpha}(0)\right]\right)=\left[P_{\beta}(0)\right]$.

Proof. We have shown $(1) \Rightarrow(2)$ in 3.1 .

Suppose (2). Note that $\mathfrak{A} \times_{\alpha} \mathbf{T}$ and $\mathfrak{A} \times_{\beta} \mathbf{T}$ are stable AF algebras and $\widehat{\alpha}_{*}, \widehat{\beta}_{*}, \varphi_{1}$ are all isomorphisms such that

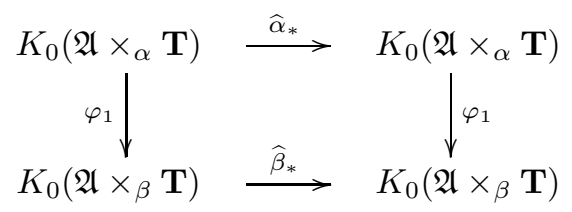

is commutative. Then by a standard intertwining argument we obtain isomorphisms $\widehat{\alpha}^{\prime}, \widehat{\beta}^{\prime}, \phi$ such that

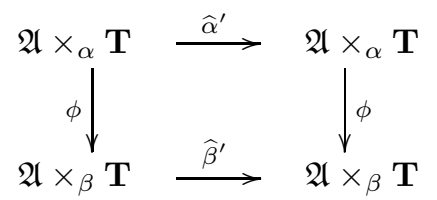

is commutative and $\widehat{\alpha}^{\prime}=\widehat{\alpha}_{*}, \widehat{\beta}^{\prime}=\widehat{\beta}_{*}$, and $\phi_{*}=\varphi_{1}$.

Since $\mathfrak{A}$ is purely infinite and $\mathfrak{A} \times{ }_{\alpha} \mathbf{T}$ has only one trace $\tau$ up to constant multiple, $\widehat{\alpha}$ must scale $\tau$, i.e. $\tau \widehat{\alpha}=\lambda \tau$ with $\lambda \neq 1$. The same is true for $\widehat{\beta}$. Hence by 1.2 , $\widehat{\alpha}^{\prime}, \widehat{\alpha}, \widehat{\beta}^{\prime}, \widehat{\beta}$ have the Rohlin property and hence $\widehat{\alpha}^{\prime}$ and $\widehat{\alpha}$ (resp. $\widehat{\beta}^{\prime}$ and $\widehat{\beta}$ ) are outer conjugate, i.e. $\widehat{\alpha}^{\prime}=\operatorname{Ad} u \sigma \widehat{\alpha} \sigma^{-1}$ and $\widehat{\beta}^{\prime}=\operatorname{Ad} v \nu \widehat{\beta} \nu^{-1}$ for unitaries $u, v$ in $\mathfrak{A} \times_{\alpha} \mathbf{T}+\mathbb{1}, \mathfrak{A} \times_{\beta} \mathbf{T}+\mathbb{1}$ and automorphisms $\sigma, \nu$ of $\mathfrak{A} \times_{\alpha} \mathbf{T}, \mathfrak{A} \times_{\beta} \mathbf{T}$, respectively. Moreover we can assume that $\sigma_{*}=$ id and $\nu_{*}=$ id. Since $\phi \widehat{\alpha}^{\prime}=\widehat{\beta}^{\prime} \phi$ we obtain

$$
(\operatorname{Ad} \phi(u)) \phi \sigma \widehat{\alpha} \sigma^{-1}=(\operatorname{Ad} v) \nu \beta \nu^{-1} \phi
$$


which implies, with $\phi_{1}=\nu^{-1} \phi \sigma$ and $u_{1}=\nu^{-1}\left(\phi\left(u^{*}\right) v\right)$,

$$
\phi_{1} \widehat{\alpha}=\operatorname{Ad} u_{1} \widehat{\beta} \phi_{1} .
$$

Let $\left\{P_{\alpha}(n) ; n \in \mathbf{Z}\right\}$ (resp. $\left.\left\{P_{\beta}(n) ; n \in \mathbf{Z}\right\}\right)$ be the spectral projections for $\lambda(t)$, $t \in \mathbf{T}$ in $\mathfrak{A} \times_{\alpha} \mathbf{T}\left(\right.$ resp. $\left.\mathfrak{A} \times_{\beta} \mathbf{T}\right)$, i.e.

$$
\lambda(t)=\sum_{n=-\infty}^{\infty} t^{n} P_{\alpha}(n) .
$$

Since $\widehat{\alpha}\left(P_{\alpha}(n)\right)=P_{\alpha}(n-1)$ and $\phi_{1 *}\left(\left[P_{\alpha}(0)\right]\right)=\left[P_{\beta}(0)\right]$, we obtain that

$$
\left[\phi_{1}\left(P_{\alpha}(n)\right)\right]=\left[P_{\beta}(n)\right] .
$$

Since $\left(\sum_{n=-N}^{N} P_{\alpha}(n)\right)_{N}$ forms an approximate identity for $\mathfrak{A} \times_{\alpha} \mathbf{T}$, there is a unitary $U$ in $M\left(\mathfrak{A} \times_{\beta} \mathbf{T}\right)$ such that $\operatorname{Ad} U \circ \phi_{1}\left(P_{\alpha}(n)\right)=P_{\beta}(n), n \in \mathbf{Z}$. Thus replacing $\phi_{1}$ by $(\operatorname{Ad} U) \phi_{1}$ we obtain an isomorphism $\phi$ of $\mathfrak{A} \times_{\alpha} \mathbf{T}$ onto $\mathfrak{A} \times_{\beta} \mathbf{T}$ such that

$$
\begin{aligned}
& \phi\left(P_{\alpha}(n)\right)=P_{\beta}(n), \quad n \in \mathbf{Z} \\
& \phi \widehat{\alpha}=(\operatorname{Ad} u) \widehat{\beta} \phi
\end{aligned}
$$

where $u$ is a unitary in $M\left(\mathfrak{A} \times_{\beta} \mathbf{T}\right)$. Since

$$
P_{\beta}(n-1)=\phi \widehat{\alpha}\left(P_{\alpha}(n)\right)=\operatorname{Ad} u\left(P_{\beta}(n-1)\right),
$$

$u$ commutes with $P_{\beta}(n)$. Let

$$
u_{n}= \begin{cases}u \widehat{\beta}(u) \ldots \widehat{\beta}^{n-1}(u), & n=1,2, \ldots \\ 1 & n=0 \\ \widehat{\beta}^{-|n|}\left(u_{|n|}^{*}\right)=\widehat{\beta}^{-1}\left(u^{*}\right) \ldots \widehat{\beta}^{-|n|}\left(u^{*}\right), & n=-1,-2, \ldots\end{cases}
$$

Then $\left\{u_{n} ; n \in \mathbf{Z}\right\}$ commutes with $P_{\beta}(n)$, and satisfies that

$$
u_{m+n}=u_{m} \widehat{\beta}^{m}\left(u_{n}\right), \quad m, n \in \mathbf{Z} .
$$

We define a unitary $v \in M\left(\mathfrak{A} \times{ }_{\beta} \mathbf{T}\right)$ by

$$
v=\sum u_{n} P_{\beta}(-n) .
$$

Then it follows that

$$
v \widehat{\beta}\left(v^{*}\right)=\sum u_{n} \widehat{\beta}\left(u_{n-1}^{*}\right) P_{\beta}(-n)=u .
$$

Hence we obtain, replacing $\left(\operatorname{Ad} v^{*}\right) \phi$ by $\phi$

$$
\phi \widehat{\alpha}=\widehat{\beta} \phi
$$

and

$$
\phi\left(P_{\alpha}(n)\right)=P_{\beta}(n) .
$$

Then the extension of $\phi$ to the multiplier algebras maps $\mathfrak{A}$ onto $\mathfrak{A}$ and

$$
\phi\left(\alpha_{t}(a)\right)=\beta_{t}(\phi(a)) \quad a \in \mathfrak{A} .
$$

(cf. Ped 7.8.8). Thus $\alpha$ is conjugate to $\beta$.

Corollary 4.2. Let $\mathfrak{A}$ be a unital separable simple purely infinite $C^{*}$-algebra and let $\alpha, \beta$ be actions of $\mathbf{T}$ on $\mathfrak{A}$ such that $\mathfrak{A} \times{ }_{\alpha} \mathbf{T}$ and $\mathfrak{A} \times{ }_{\beta} \mathbf{T}$ are simple AF algebras with one-dimensional lattice of traces. Then the following conditions are equivalent:

1. $\alpha$ and $\beta$ are cocycle conjugate; 
2. $\left(K_{0}\left(\mathfrak{A} \times_{\alpha} \mathbf{T}\right),\left[P_{\alpha}(0)\right]+\operatorname{Im}\left(\mathrm{id}-\widehat{\alpha}_{*}\right), \widehat{\alpha}_{*}\right)$ and $\left(K_{0}\left(\mathfrak{A} \times{ }_{\beta} \mathbf{T}\right),\left[P_{\beta}(0)\right]+\operatorname{Im}\left(\mathrm{id}-\widehat{\beta}_{*}\right)\right.$, $\left.\widehat{\beta}_{*}\right)$ are isomorphic, i.e., there is an order isomorphism $\varphi_{1}$ of $K_{0}\left(\mathfrak{A} \times_{\alpha} \mathbf{T}\right)$ onto $K_{0}\left(\mathfrak{A} \times_{\beta} \mathbf{T}\right)$ such that $\varphi_{1} \widehat{\alpha}_{*}=\widehat{\beta}_{*} \varphi_{1}$ and $\varphi_{1}\left(\left[P_{\alpha}(0)\right]\right)-\left[P_{\beta}(0)\right] \in \operatorname{Im}\left(\operatorname{id}-\widehat{\beta}_{*}\right)$.

Proof. We have shown (1) $\Rightarrow(2)$ in 3.2 .

Assume that (2) holds. As in the proof of 4.1 we obtain an isomorphism $\phi$ of $\mathfrak{A} \times_{\alpha} \mathbf{T}$ onto $\mathfrak{A} \times_{\beta} \mathbf{T}$ such that

$$
\phi \widehat{\alpha}=(\operatorname{Ad} u) \widehat{\beta} \phi
$$

for some unitary $u \in \mathfrak{A} \times_{\beta} \mathbf{T}+\mathbb{1}$.

Since $\left(\mathfrak{A} \times{ }_{\beta} \mathbf{T} \times(\operatorname{Ad} u) \widehat{\beta} \mathbf{Z}, \mathbf{T},((\operatorname{Ad} u) \widehat{\beta})\right)$ is isomorphic to $\left(\mathfrak{A} \times{ }_{\beta} \mathbf{T} \times{ }_{\widehat{\beta}} \mathbf{Z}, \mathbf{T}, \widehat{\widehat{\beta}}\right)$, which is again isomorphic to $\left(\mathfrak{A} \otimes \mathfrak{K}\left(L^{2}(\mathbf{T})\right), \mathbf{T}, \beta \otimes \operatorname{Ad} \widetilde{\lambda}\right)$, we obtain an isomorphism $\widehat{\phi}$ of $\mathfrak{A} \otimes \mathfrak{K}\left(L^{2}(\mathbf{T})\right)$ onto $\mathfrak{A} \otimes \mathfrak{K}\left(L^{2}(\mathbf{T})\right)$ such that $\widehat{\phi}\left(\alpha_{t} \otimes \operatorname{Ad} \widetilde{\lambda}_{t}\right)=\left(\beta_{t} \otimes \operatorname{Ad} \widetilde{\lambda}_{t}\right) \widehat{\phi}$, extending $\phi: \mathfrak{A} \times_{\alpha} \mathbf{T} \rightarrow \mathfrak{A} \times_{\beta} \mathbf{T}$. The image of $\left[P_{\alpha}(0)\right] \in K_{0}\left(\mathfrak{A} \times_{\alpha} \mathbf{T}\right)$ in $K_{0}(\mathfrak{A} \otimes$ $\mathfrak{K}\left(L^{2}(\mathbf{T})\right)=K_{0}(\mathfrak{A})$ is $[\mathbb{1}]$; and $\widehat{\phi}_{*}$ preserves this class, i.e., $[\widehat{\phi}(\mathbb{1} \otimes p)]=[\mathbb{1} \otimes p]$ where $p$ is a minimal projection in $\mathfrak{K}\left(L^{2}(\mathbf{T})\right)$.

Thus we find a unitary multiplier $U$ of $\mathfrak{A} \otimes \mathfrak{K}\left(L^{2}(\mathbf{T})\right)$ such that $(\operatorname{Ad} U \widehat{\phi})$ is the identity on $\mathbb{1} \otimes \mathfrak{K}\left(L^{2}(\mathbf{T})\right)$. Then there is an automorphism $\sigma$ of $\mathfrak{A}$ such that $(\operatorname{Ad} U) \widehat{\phi}=\sigma \otimes$ id, from which it follows that

$$
\sigma \alpha_{t} \otimes \mathrm{id}=\operatorname{Ad} U\left(\beta_{t} \otimes \operatorname{Ad} \widetilde{\lambda}_{t}\right)\left(U^{*}\right) \beta_{t} \sigma \otimes \mathrm{id} .
$$

Using $u_{t}=U\left(\beta_{t} \otimes \operatorname{Ad} \tilde{\lambda}_{t}\right)\left(U^{*}\right) \in \mathfrak{A}$, which is a one-cocycle for $\beta$, we obtain that

$$
\sigma \alpha_{t}=\operatorname{Ad} u_{t} \beta_{t} \sigma \text {. }
$$

This shows that $\alpha$ is cocycle conjugate to $\beta$.

If $\mathfrak{A}$ is not unital we still have the following:

Corollary 4.3. Let $\mathfrak{A}$ be a separable simple purely infinite $C^{*}$-algebra and let $\alpha, \beta$ be actions of $\mathbf{T}$ on $\mathfrak{A}$ such that $\mathfrak{A} \times_{\alpha} \mathbf{T}$ and $\mathfrak{A} \times_{\beta} \mathbf{T}$ are simple AF algebras with one-dimensional lattice of traces. Then the following conditions are equivalent:

1. $\left(\mathfrak{A} \otimes \mathfrak{K}\left(L^{2}(\mathbf{T})\right), \mathbf{T}, \alpha \otimes \mathrm{id}\right)$ is isomorphic to $\left(\mathfrak{A} \otimes \mathfrak{K}\left(L^{2}(\mathbf{T})\right), \mathbf{T}, \beta \otimes \mathrm{id}\right)$

2. $\left(\mathfrak{A} \otimes \mathfrak{K}\left(L^{2}(\mathbf{T})\right), \mathbf{T}, \alpha \otimes \operatorname{Ad} \widetilde{\lambda}\right)$ is isomorphic to $\left(\mathfrak{A} \otimes \mathfrak{K}\left(L^{2}(\mathbf{T})\right), \mathbf{T}, \beta \otimes \operatorname{Ad} \widetilde{\lambda}\right)$

3. $\left(K_{0}\left(\mathfrak{A} \times_{\alpha} \mathbf{T}\right), \widehat{\alpha}_{*}\right)$ is isomorphic to $\left(K_{0}\left(\mathfrak{A} \times_{\beta} \mathbf{T}\right), \widehat{\beta}_{*}\right)$.

Proof. By general theory we obtain that $(1) \Rightarrow(3)$ and $(2) \Rightarrow(3)$. From a part of the proof of 4.2 we obtain $(3) \Rightarrow(2)$.

Assume that (3) holds. Denoting by $\mathfrak{K}$ the compact operators on $L^{2}(\mathbf{T})$, we consider the systems $(\mathfrak{A} \otimes \mathfrak{K}, \mathbf{T}, \alpha \otimes$ id $)$ and $(\mathfrak{A} \otimes \mathfrak{K}, \mathbf{T}, \beta \otimes$ id $)$. Since $\mathfrak{A}^{\alpha}$ is a hereditary $\mathrm{C}^{*}$-subalgebra of $\mathfrak{A} \times{ }_{\alpha} \mathbf{T}$ and $\mathfrak{A} \times{ }_{\alpha} \mathbf{T}$ is a stable simple AF algebra, we have that $\mathfrak{A}^{\alpha} \otimes \mathfrak{K} \cong \mathfrak{A} \times_{\alpha} \mathbf{T}$. In the spectral subspace

$$
\left\{x \in M(\mathfrak{A} \otimes \mathfrak{K}):\left(\alpha_{z} \otimes \mathrm{id}\right)(x)=z x, z \in \mathbf{T}\right\}
$$

we find a unitary $U$ [KT]. Note that $\mathfrak{A} \otimes \mathfrak{K}$ is generated by $\mathfrak{A}^{\alpha} \otimes \mathfrak{K}$ and $U \mathfrak{A}^{\alpha} \otimes \mathfrak{K}$. Define an automorphism $\gamma$ of $\mathfrak{A}^{\alpha} \otimes \mathfrak{K}$ by $\gamma=\operatorname{Ad} U$.

Then, as $\widehat{\gamma}=\alpha \otimes \mathrm{id},\left(\mathfrak{A}^{\alpha} \otimes \mathfrak{K} \times{ }_{\gamma} \mathbf{Z} \times \widehat{\gamma} \mathbf{T}, \widehat{\gamma}\right)$ is isomorphic to $\left(\left(\mathfrak{A} \times{ }_{\alpha} \mathbf{T}\right) \otimes \mathfrak{K}, \widehat{\alpha} \otimes \mathrm{id}\right)$, which implies that $\left(K_{0}\left(\mathfrak{A}^{\alpha} \otimes \mathfrak{K}\right), \gamma_{*}\right) \cong\left(K_{0}\left(\mathfrak{A} \times_{\alpha} \mathbf{T}\right), \widehat{\alpha}_{*}\right)$.

In the same way we obtain a unitary $V$ in

$$
\left\{x \in M(\mathfrak{A} \otimes \mathfrak{K}) ; \beta_{z} \otimes \operatorname{id}(x)=z x, z \in \mathbf{T}\right\}
$$


and that

$$
\left(K_{0}\left(\mathfrak{A}^{\beta} \otimes \mathfrak{K}\right),(\operatorname{Ad} V)_{*}\right) \cong\left(K_{0}\left(\mathfrak{A} \times_{\beta} \mathbf{T}\right), \widehat{\beta}_{*}\right) .
$$

Hence, by using the Rohlin property for $\operatorname{Ad} U \mid \mathfrak{A}^{\alpha} \otimes \mathfrak{K}$ and $\operatorname{Ad} V \mid \mathfrak{A}^{\beta} \otimes \mathfrak{K}$, we obtain an isomorphism $\varphi$ of $\mathfrak{A}^{\alpha} \otimes \mathfrak{K}$ onto $\mathfrak{A}^{\beta} \otimes \mathfrak{K}$ such that

$$
\varphi \circ \operatorname{Ad} U=\operatorname{Ad} v V \circ \varphi
$$

for some unitary $v \in \mathfrak{A}^{\beta} \otimes \mathfrak{K}+\mathbb{1}$. Then $\varphi$ extends to an isomorphism $\widehat{\varphi}$ of $\mathfrak{A} \otimes \mathfrak{K}$ onto $\mathfrak{A} \otimes \mathfrak{K}$ such that

$$
\begin{aligned}
& x \mapsto \varphi(x), \\
& U x \mapsto v V x
\end{aligned}
$$

for $x \in \mathfrak{A}^{\alpha} \otimes \mathfrak{K}$. It follows that $\widehat{\varphi}(\alpha \otimes$ id $)=(\beta \otimes$ id $) \widehat{\varphi}$.

A class of examples of actions of $\mathbf{T}$ on Cuntz algebras $\mathcal{O}_{d}$ (with $d<\infty$ ) is obtained from quasi-free actions [BJO]. Other examples, which we consider in Example 4.4 and 4.5 below, are obtained starting with trace-scaling automorphisms of AF algebras.

Example 4.4. For each $\lambda \in(0,1) \cup(1, \infty)$ we define an order on $\mathbf{Z}\left[x, x^{-1}\right]$ by $p \geq q$ if $p\left(\lambda, \lambda^{-1}\right)>q\left(\lambda, \lambda^{-1}\right)$ or $p \equiv q$. Denote the ordered abelian group so obtained by $\mathbf{Z}\left[x, x^{-1}\right]_{\lambda}$. Since $\mathbf{Z}\left[\lambda, \lambda^{-1}\right]$ is dense in $\mathbf{R}, \mathbf{Z}\left[x, x^{-1}\right]_{\lambda}$ is a dimension group. Let $\mathfrak{A}_{\lambda}$ be the stable AF algebra whose dimension group is isomorphic to $\mathbf{Z}\left[x, x^{-1}\right]_{\lambda}$ and $\alpha$ an automorphism of $\mathfrak{A}_{\lambda}$ such that $\alpha_{*}$ corresponds to the multiplication by $x$. Then $\mathfrak{A}_{\lambda} \times_{\alpha} \mathbf{Z}$ is purely infinite [R2] and it is isomorphic to $\mathcal{O}_{\infty} \otimes \mathfrak{K}$ Phi since $K_{0}\left(\mathfrak{A}_{\lambda} \times_{\alpha} \mathbf{Z}\right)=\mathbf{Z}\left[x, x^{-1}\right] /(1-x) \mathbf{Z}\left[x, x^{-1}\right] \cong \mathbf{Z}$. By cutting down by a projection $p \in \mathfrak{A}_{\lambda}$ with $[p]=1 \in \mathbf{Z}\left[x, x^{-1}\right]_{\lambda}$, we thus obtain an action $\gamma_{\lambda}$ on $\mathcal{O}_{\infty} \cong p\left(\mathfrak{A}_{\Lambda} \times_{\alpha} \mathbf{Z}\right) p$ from the dual action $\widehat{\alpha}$. The invariant for the cocycle conjugacy class of $\left(\mathcal{O}_{\infty}, \mathbf{T}, \gamma_{\lambda}\right)$ is given by

$$
\left(\mathbf{Z}\left[x, x^{-1}\right]_{\lambda}, 1+(1-x) \mathbf{Z}\left[x, x^{-1}\right]_{\lambda}, x\right)
$$

Note that each $\gamma_{\lambda}$ has a unique KMS state at the inverse temperature $-\ln \lambda$.

Example 4.5. For $d=1,2, \ldots$ denote by $G$ the abelian group

$$
\mathbf{Z}\left[x, x^{-1}\right]+d \mathbf{Z}\left[x, x^{-1},(1-x)^{-1}\right]
$$

and for $\lambda \in(0,1) \cup(1, \infty)$, denote by $G_{\lambda}$ the ordered abelian group $G$ with the order defined by: $p \geq 0$ if $p\left(\lambda, \lambda^{-1},(1-\lambda)^{-1}\right)>0$ or $p \equiv 0$. Denote by $\mathfrak{A}_{\lambda}$ the stable AF algebra corresponding to $G_{\lambda}$ and by $\alpha$ an automorphism of $\mathfrak{A}_{\lambda}$ corresponding to the multiplication by $x$. Then $\alpha$ has the Rohlin property. Since

$$
G /(1-x) G \cong \mathbf{Z} / d \mathbf{Z}
$$

$\mathfrak{A}_{\lambda} \times_{\alpha} \mathbf{Z}$ is isomorphic to $\mathcal{O}_{d+1} \otimes K$. By cutting down $\mathfrak{A}_{\lambda} \times_{\alpha} \mathbf{Z}$ by a projection $p \in \mathfrak{A}_{\lambda}$ with $[p]=1$ we obtain an action $\gamma_{\lambda}$ of $\mathbf{T}$ on $\mathcal{O}_{d+1}$ from the dual action $\widehat{\alpha}$. Thus we obtain a one parameter family $\left(\mathcal{O}_{d+1}, \mathbf{T}, \gamma_{\lambda}\right)$ of actions of $\mathbf{T}$ on $\mathcal{O}_{d+1}$ just for $\mathcal{O}_{\infty}$ in the previous example.

\section{REFERENCES}

[Bla] Blackadar, B.: Symmetries of the CAR algebra, Annals Math. 131 (1990), 589-623.

[Bra72] Bratteli, O.: Inductive limits of finite dimensional $\mathrm{C}^{*}$-algebras, Trans. Amer. Math. Soc. 171 (1972), 195-234.

[BEH] Bratteli, O., Elliott, G.A., Herman, R.H.: On the possible temperatures of a dynamical system, Comm. Math. Phys. 74 (1980), 281-295. 
[BJO] Bratteli, O., Jorgensen, P.E.T., Ostrov'sky, V.: The representations and centralizers of certain states on $\mathcal{O}_{d}$, in preparation.

[C74] Connes, A.: Almost periodic states and factors of type $\mathrm{III}_{1}$, J. Funct. Anal. 16 (1974), $415-445$.

[C75] Connes, A.: Outer conjugacy classes of automorphisms of factors, Ann. Scient. Ec. Norm. Sup. 4 serie, 8 (1975) 383-420.

[Eff] Effros, E.G.: Dimensions and $\mathrm{C}^{*}$-algebras, CBMS regional conference series in Mathematics, vol. 46, AMS, 1981.

[EEK] Elliott, G.E., Evans, D.E., Kishimoto, A.: Outer conjugacy classes of trace scaling automorphisms of stable UHF algebras, Math. Scand. 83 (1998), 74-86.

[EK] Evans, D.E., Kishimoto, A.: Trace scaling automorphisms of certain stable AF algebras, Hokkaido Math. J. 26 (1997), 211-224.

[Fer] Ferguson, Le Baron O.: Approximation of polynomials with integral coefficients, AMS, 1980.

[HR] Handelman, D., Rossmann, W.: Actions of compact groups on AF algebras, Illinois J. of Math. 29 (1985), 51-95.

[KP] Kirchberg, E., Phillips, N.C.: Embedding of exact $\mathrm{C}^{*}$-algebras and continuous fields in the Cuntz algebra $\mathcal{O}_{2}$, preprint.

[K95] Kishimoto, A.: The Rohlin property for automorphisms of UHF algebras, J. reine angew. Math. 465 (1995), 183-196.

[K96] Kishimoto, A.: The Rohlin property for shifts on UHF algebras and automorphisms of Cuntz algebras, J. Funct. Anal. 140 (1996), 100-123.

[K98] Kishimoto, A.: Unbounded derivations in AT algebras, J. Funct. Anal. 160 (1998), $270-311$.

[KT] Kishimoto, A., Takai, H.: Some remarks on $\mathrm{C}^{*}$-dynamical systems with a compact abelian group, Publ. RIMS, Kyoto Univ. 14 (1978), 383-397.

[Kri] Krieger, W.: On dimension functions and topological Markov chains, Invent. Math. 56 (1980), 239-250.

[Nak] Nakamura, H.: Aperiodic automorphisms of nuclear purely infinite simple $\mathrm{C}^{*}$-algebras, preprint.

[Ped] Pedersen, G.K.: C*-algebras and their automorphism groups, Academic Press, 1979.

[Phi] Phillips, N.C.: A classification theorem for nuclear purely infinite simple $\mathrm{C}^{*}$-algebras.

[R1] Rørdam, M.: Classification of certain infinite simple $\mathrm{C}^{*}$-algebras, J. Funct. Anal. 131 (1995), 415-458.

[R2] Rørdam, M.: Classification of certain infinite simple $\mathrm{C}^{*}$-algebras, III, Fields Inst. Commun. 13 (1997), 257-282.

[Tak] Takai, H.: On a duality for crossed products of $\mathrm{C}^{*}$-algebras, J. Funct. Anal. 19 (1975), 25-39.

[Tor] Török, A.: AF-algebras with unique trace, Acta Sci. Math. (Szeged) 55 (1991), 129-139.

Mathematics Institute, University of Oslo, PB 1053 Blindern, N-0316 Oslo, Norway

Department of Mathematics, Hokkaido University, Sapporo, 060 Japan 